\title{
CLASSIFICATION OF INJECTIVE FACTORS: THE WORK OF ALAIN CONNES
}

\author{
STEVE WRIGHT \\ Department of Mathematical Sciences \\ Oakland University \\ Rochester, Michigan 48063 \\ (Received May 7, 1981)
}

ABSTRACT: The fundamental results of A. Connes which determine a complete set of isomorphism classes for most injective factors are discussed in detail. After some introductory remarks which lay the foundation for the subsequent discussion, an historical survey of some of the principal lines of the investigation in the classification of factors is presented, culminating in the Connes-Takesaki structure theory of type III factors. After a discussion of injectivity for finite factors, the main result of the paper, the uniqueness of the injective $\mathrm{II}_{1}$ factor, is deduced, and the structure of II $_{\infty}$ and type III injective factors is then obtained as corollaries of the main result.

1980 AMS (MOS) SUBJECT CLASSIFICATION: PRIMARY - 46L35, 46L10;

SECONDARY - 46L50, 46LO5

KEY WORDS AND PHRASES: injective von Neumann algebra, finite factor, discrete and continuous crossed products, infinite tensor product, semidiscrete von Neumann algebra.

I. INTRODUCTION.

From the beginning, one of the central problems of the theory of von Neumann algebras has been their classification according to isomorphism type. The attack on the problem was initiated by the founding fathers Murray and von Neumann $([1],[2],[3],[4])$, who introduced the fundamental notion of types $\left(I, I_{1}, I_{\infty}\right.$, and III), and who claimed early victories with the characterization of the hyperfinite 
$\mathrm{II}_{1}$ factor and a complete classification of the factors of type I. As the years passed, there was vigorous development of the theory, but no results appeared that were as definitive as these early advances. However, in 1973 a new era dawned with the publication of the thesis [5] of Alain Connes. Basing his work on earlier results of Tomita and Takesaki [6], Connes initiated a program for the classification of factors that can be termed as nothing short of revolutionary. In [5], [7], [8], and [9], he obtained a classification of factors of type III and automorphisms of certain factors of type $\mathrm{II}_{1}$ and $\mathrm{II}_{\infty}$ which culminated in the remarkable work of [10], in which appear the first major advances beyond the classical theory in the classification of factors for the non-type I case. The purpose of these notes is to give a rather detailed discussion of the most important of these results.

We assume the reader has a familiarity with the theory of von Neumann algebras on the level of [11], say. We recall some basic facts that play an important role in the sequel. A factor is a von Neumann algebra with a trivial center, i.e., the only elements of the algebra which commute with every element are scalar multiples of the identity. We will be concerned primarily with von Neumann algebras of finite type, and we will use the tracial characterization of this (see [12], Section III-8). Recall that a trace $\tau$ on the factor $\mathrm{N}$ is a positive linear functional of norm 1, i.e., a state on $N$, which satisfies

$$
\tau(\mathrm{ab})=\tau(\mathrm{ba}), \forall \mathrm{a}, \mathrm{b} \in \mathrm{N} \text {. }
$$

A factor is said to be finite if it has a trace. The trace on a finite factor is uniquely determined among the states by (1.1), and it is automatically faithful and ultraweakly continuous (Theorem 2.4.6 of [13]). These facts will be used frequent1y.

We will also employ the standard representation of a finite factor $\mathrm{N}$. Let $\tau$ be the (canonical) trace on $N$. Then the representation $\pi_{\tau}$ in the GelfandNaimark-Segal construction $\left\{\pi_{\tau}, \mathrm{H}_{\tau}, \xi_{\tau}\right\}$ corresponding to $\tau$ is faithful and ultraweakly continuous, and the cyclic vector $\xi_{\tau}$ is separating for $\pi_{\tau}(\mathrm{N})$ (i.e., $\xi_{\tau}$ is cyclic for $\pi_{\tau}(\mathrm{N})^{\prime}$, where ' denotes the commutant). We denote $\mathrm{H}_{\tau}$ by $L^{2}(N, \tau)$, and the $\underline{L}^{2}$-norm of $x \in N$ is by definition $\|x\|_{2}=\tau\left(x^{*} x\right)^{\frac{1}{2}}$. $\pi_{\tau}$ is 
called the standard representation of $N$, and when we identify $N$ with its image $\pi_{\tau}(N)$ in $H=L^{2}(N, \tau)$, we will say that $N$ acts standardly in $H$. Tensor products of C*-algebras and von Neumann algebras wi.11 play an important role in what follows. Let $A_{1}, i=1,2$ be $C^{*}$-algebras, which for convenience we will assume act on Hilbert spaces $H_{i}, i=1,2$. Form the algebraic tensor product $A_{1} \Theta A_{2}$, which can be viewed in a natural way as a $\star$-subalgebra of $\mathrm{B}\left(\mathrm{H}_{1} \otimes \mathrm{H}_{2}\right)$ the algebra of bounded operators on $\mathrm{H}_{1} \otimes \mathrm{H}_{2}$. A seminorm $\rho$ on $A_{1} \otimes A_{2}$ is called a C*-subcross seminorm if

(i) $\rho\left(x^{*} x\right)=\rho(x)^{2}, x \in A_{1} \odot A_{2}$,

(ii) $\rho\left(a_{1} \otimes a_{2}\right) \leq\left\|a_{1}\right\|\left\|a_{2}\right\|, a_{i} \in A_{i}, i=1,2$.

$\rho$ is a c*-crossnorm if $\rho$ is a norm satisfying ( $i$ ), and (ii) with $\leq$ replaced by $=$. If we take the supremum of all $\mathrm{C} *$-subcross seminorms defined on $A_{1} \bullet A_{2}$ we will obtain a $C^{*}$-crossnorm $\nu$ which by definition will be the 1zrgest $C^{*}$-crossnorm definable on $A_{1} \odot A_{2}$. Another crossnorm can be defined on $A_{1} \odot A_{2}$ by recalling that $A_{1} \otimes A_{2}$ acts on $H_{1} \otimes H_{2}$, and so each element of $A_{1} \Theta A_{2}$ has a norm II $\|$ considered as a boumded operator on $\mathrm{H}_{1} \otimes \mathrm{H}_{2}$. It can be shown that the operator norm \|\| is the smallest $C *$-crossnorm definable on $A_{1} \Theta A_{2}$. Completing $A_{1} \odot A_{2}$ in the $\nu$-norm and the \|\| -norm will yield C*-algebras which we denote respectively by $A_{1} \otimes_{\max } A_{2}$ and $A_{1} \otimes_{\min } A_{2}$, the so-called maximal and minimal C*-tensor products of $A_{1}$ and $A_{2}$. These algebras will in general be distinct. If we further assume that $A_{1}$ and $A_{2}$ are von Neumann algebras, we may also take the closure of $A_{1} \odot A_{2}$ relative to the weak operator topology on $B\left(\mathrm{H}_{1} \otimes \mathrm{H}_{2}\right)$, and thus obtain a von Neumann subalgebra of $B\left(\mathrm{H}_{1} \otimes \mathrm{H}_{2}\right)$, the (spatial) $W^{*}$-tensor product $A_{1} \otimes A_{2}$ of $A_{1}$ and $A_{2} \cdot$ An excellent source of information about tensor products is [14].

Suppose $M_{i}$ and $N_{i}$ are finite factors, $i=1,2$, and $M_{i} \cong N_{i}, i=1,2$ $\left(\cong\right.$ denotes isomorphism). We claim that $M_{1} \otimes M_{2} \cong N_{1} \otimes N_{2}$. Let $\varphi_{i}: M_{i} \rightarrow N_{i}$ be isomorphisms, and let $\tau_{i}, \sigma_{i}$ denote the canonical traces on $M_{i}$ and $N_{i}$, respectively. By uniqueness of the trace,

$$
\sigma_{i} \circ \varphi_{i}=\tau_{i}, \quad i=1,2 .
$$


Now $\varphi_{1} \otimes \varphi_{2}$ is an isomorphism of $M_{1} \otimes M_{2}$ onto $N_{1} \ominus N_{2}$, and by (1.2), $\left(\sigma_{1} \otimes \sigma_{2}\right) \circ\left(\varphi_{1} \otimes \varphi_{2}\right)=\tau_{1} \otimes \tau_{2}$. Since $\sigma_{1} \otimes \sigma_{2}$ and $\tau_{1} \otimes \tau_{2}$ are the canonical traces on $N_{1} \otimes N_{2}$ and $M_{1} \otimes M_{2}$, respectively, they are faithful and normal. Thus by [15], Lemma 1, (see Lemma 9.2 infra) $\varphi_{1} \otimes \varphi_{2}$ extends to an isomorphism of $M_{1} \otimes M_{2}$ onto $N_{1} \otimes N_{2}$.

Finally, we define the infinite tensor product of von Neumann algebras. Let $\left\{M_{n}\right\}$ be a sequence of von Neumann algebras, let $\varphi_{n}$ be a normal state on $M_{n}$, and let $\varphi=\otimes_{\mathbf{n}}^{\otimes} \varphi_{\mathrm{n}}$ denote the infinite tensor product state on the infinite C*-tensor product $\underset{n}{\otimes} M_{n}$ of the $M_{n}^{\prime} ' s$ ([13], Section 1.23). Let $\pi_{\varphi}$ denote the Gelfand-Naimark-Segal representation of $\otimes_{n}$ determined by $\varphi$. Then the von Neumann algebra $\pi_{\varphi}\left(\otimes_{n} M_{n}\right) "$ is called the $\left(W^{*-}\right)$ infinite tensor product of $\left\{M_{n}\right\}$ relative to $\varphi$.

2. AN HISTORICAL PERSPECTIVE ON CONNES' WORK

We will preface our discussion of [10] by a rather brief overview of the main lines of work which led up to it. Because of the limitations of space and time, we have concentrated on emphasizing only work which deals directly with the classification of factors, and have reluctantly suppressed discussion of many other interesting and important developments in the structure theory of von Neumann algebras.

Our story begins, as all stories about von Neumann algebras do, with the work of Murray and von Neumann [1], [2], [3], and [4]. The classification of factors was the motivating problem for this work, and all other progress on the problem was based on this pioneering effort. In [1], Murray and von Neumann introduced the fundamental notion of types (I, II,$I_{\infty}$, and III), and in [1] and [2], they obtained a complete classification of the factors of type I: if $M$ is a factor of type $I$, then there exists a Hilbert space $H$ such that $M$ is isomorphic to $B(H)$, the algebra of all bounded operators on $\mathrm{H}$. For our purposes, however, the most important advance that Murray and von Neumann made was their famous characterization of the hyperfinite $\mathrm{II}_{1}$ factor. Since this result will play a key role in the sequel, we will describe it in detail. 
Let $M$ be a von Neumann algebra acting on a separable Hilbert space (from now antil the end of this section, all von Neumann algebras will be assumed acting on a separable Hilbert space without explicit mention). $M$ is said to be hyperfinite if there is a sequence $\left\{M_{n}\right\}$ of finite-dimensional von Neumann subalgebras of $M$, totally ordered by inclusion, whose union is dense in $M$ relative to the weak operator topology. If $M$ is a finite factor, hyperfiniteness of $M$ is equivalent to the following condition (condition $\underline{C}$ of Murray-von Neumann): for each finite subset $\left\{x_{1}, \ldots, x_{n}\right\}$ of $M$ and $\varepsilon>0$, there is a finite-dimensional subfactor $C$ of $M$ and $v_{1}, \ldots, v_{n} \in C$ such that $\left\|x_{i}-v_{i}\right\|_{2}<\varepsilon, i=1, \ldots, n$. The following theorem will be used in a crucial way in our later discussion:

2.1. THEOREM. ([4], Theorem XIV). Let $M_{1}$ and $M_{2}$ be faistors of type $I_{1}$. If $M_{1}$ and $M_{2}$ both satisfy condition $C$, then $M_{1}$ and $M_{2}$ are isomorphic.

We will denoce by $R$ the hyperfinite $I_{1}$ factor, unique up to isomorphism by Theorem 2.1.

Another important technique contained in the Murray-von Neumann parers is the so-called group-measure space construction of factors. This technique was used to give the first examples of type $I I_{1}$ and $I I_{\infty}$ factors in [2], and the first example of a factor of type III in [3]. We will not go into the details of this construction right now: it will emerge later as a special case of the ConnesTakesaki crossed product construction.

The next major advance in the structure theory of von Neumann algebras occured in 1949 with the publication of von Neumann's reduction theory [16]. This paper intraduced and used the concept of direct integral of Hilbert spaces to decompose an arbitrary separably acting von Neumann algebra into a direct integral of factors, and thereby reduced the study of von Neumann algebras, at least in principle, to the study of factors.

This then was essentially the state of the art in the classification of von Neumann algebras at the start of the $1950^{\prime} \mathrm{s}$. Most of the work in the area consisted in a refining and strengthening of the tools bequeathed by Murray and 
von Neumann. The theory at that time suffered from a dearth of examples of nonisomorphic factors, and consequently one of the main lines of work was the construction of such examples. Von Neumann had constructed one example of a type III factor in [3], but did not give another nonisomorphic one. In 1956, Pukansky [17] constructed a pair of nonisomorphic type III factors using refinements of the group-measure space construction, and there the matter lay until 1963 when J.T. Schwartz [18] found a new invariant, his well-known Property $P$ : a von Neumann algebra $M$ acting on $H$ has Property $P$, if for each $T \in B(H)$, the weakly closed convex hull of $\left\{\mathrm{U} T \mathrm{U}^{*}\right.$ : U a unitary operator in M\} has a nonempty intersection with $M^{\prime}$. Schwartz used this property to distinguish two nonisomorphic, hyperfinite $\mathrm{II}_{1}$ factors, and also to construct another type III factor different from Pukansky's examples [19]. He showed that all hyperfinite factors have property $\mathrm{P}$, and conjectured the converse. This converse was one of the many things established by Connes in [10].

Since it had been a fairly difficult task to obtain such a small number of examples of nonisomorphic factors, there gradually emerged a hope that a somewhat complete classification of all factors just might be possible. In 1967, that hope began to fade when R.T. Powers [20] constructed the first uncountable family of nonisomorphic factors. Let $\lambda \in[0,1]$ and let $M_{2}$ denote the $2 \times 2$ complex matrices. Let $\varphi_{\lambda}$ denote the state defined on $M_{2}$ defined by

$$
\varphi_{\lambda}:\left[\begin{array}{ll}
\alpha & \beta \\
\gamma & \delta
\end{array}\right] \rightarrow \frac{\lambda}{1+\lambda} \alpha+\frac{1}{1+\lambda} \delta
$$

The Powers factor $R_{\lambda}$ is defined as the infinite tensor product of countably many copies of $M_{2}$ relative to the product state $\underset{n}{\otimes} \varphi_{n}$, where each $\varphi_{n}$ is $\varphi_{\lambda}$. We note that $R_{1}$ is the hyperfinite $I I_{1}$ factor. $R_{\lambda}$ is a hyperfinite factor of type III for each $\lambda \in(0,1)$, and Powers showed that $R_{\lambda_{1}}$ is not isomorphic to $R_{\lambda_{2}}$ if $\lambda_{1}$ and $\lambda_{2}$ are distinct elements of $(\theta, 1)$. We will see this class of factors again.

After Powers' examples appeared, the flood gates opened, and nonisomorphic 
factors soon poured out. In 1969, McDuff [21] exhibited an uncountable family of II $_{1}$ factors, and in 1970, Sakai [22], [23] found uncountable families of II and nonhyperfinite type III factors. This work dashed forever the hope of a complete classification of all factors. Attention began to focus on particular classes of factors for which there did seem to be a possibility of a satisfactory classification with results that were to shortly revolutionize the theory.

Let $\left\{M_{n}\right\}$ be a sequence of finite factors, with $M_{n}$ of type $I_{m_{n}}, m_{n}<\infty$, for each $n$. Assume that $M_{n}$ acts on $H_{n}$, and let $x_{n}$ be a fixed unit vector in $H_{n}$. Then the functional $\omega_{x_{n}}$ defined on $M_{n}$ by $\omega_{x_{n}}: T \rightarrow\left(T x_{n}, x_{n}\right)$, $T \in M_{n}$ is a state on $M_{n}$. Form the infinite tensor product $\underset{n}{\otimes} M_{n}$ relative to the product state $\underset{\mathrm{n}}{\otimes} \omega_{\mathrm{x}_{\mathrm{n}}}$. The resulting factor is an example of what Araki and Woods [24] dubbed an ITPFI factor (for "infinite tensor product of finite type I's"). In this paper, which appeared in 1968 and was motivated in part by Powers' work, these authors introduced an important new invariant for the ITPFI factors, the asymtotic ratio set, and succeeded in obtaining an almost complete classification of this class of factors. Both for its historical importance and for its foreshadowing of even bigger things to come, we will briefly describe the asymptotic ratio set and the Araki-Woods classification.

Let $M$ be a factor. The asymptotic ratio set $r_{\infty}(M)$ of $M$ is the subset of $[0, \infty)$ defined as follows:

$$
\begin{aligned}
r_{\infty}(M) & =\left\{x \in[0,1]: M \text { is isomorphic to } M \otimes R_{x}\right\} \\
& U\left\{x \in(1, \infty): M \text { is isomorphic to } M \otimes R_{1 / x}\right\}
\end{aligned}
$$

where $R_{\lambda}$ is the Powers factor defined previously. This is not the definition of $r_{\infty}(M)$ first given by Araki and Woods for the ITPFI factors. The first definition ([24], Definition 3.2) was expressed in terms of a complicated limiting procedure involving the ratios of the eigen-values of the density matrices of the states occurring in the infinite tensor product decomposition of the ITPFI factors, and Araki and Woods later showed that the definition given above was equivalent to the original one ([24], proof of Theorem 5.9). If $M$ is an ITPFI factor, they deduced that $r_{\infty}(M)$ must have one of the following forms: 


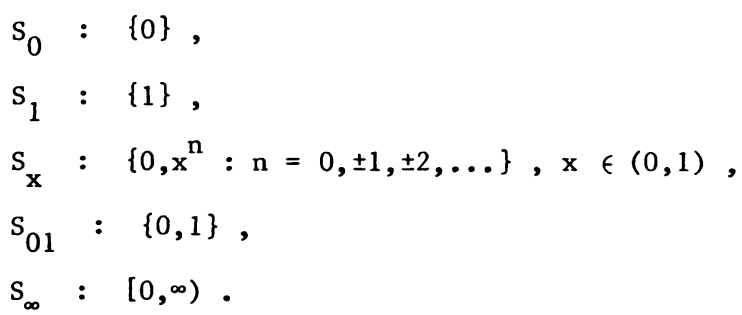

Araki and Woods showed that $S_{\infty}$ corresponds to only one isomorphism class of ITPFI factors, and it is of type III. We will denote this factor by $R_{\infty} \cdot$ The invariants $s_{x}, x \in[0,1]$ were also shown to come from only one isomorphism class, the one determined by the Powers factor $R_{x^{*}}$ Thus all isomorphism classes of ITPFI factors, with the exception of those in the $\mathrm{S}_{01}$ class, were completely determined. The Araki-Woods classification scheme was the first significant advance in the classification of factors beyond the initial results of Murray and von Neumann, and it was to have a great influence on the young Alain Connes. The second major development which Connes would put to good use came from Japan. A problem of interest at the time concerned the commutant of a tensor product of von Neumann algebras: if $M_{1}$ and $M_{2}$ are von Neumann algebras, does $\left(M_{1} \otimes M_{2}\right)^{\prime}$ equal $M_{1}^{\prime} \otimes M_{2}^{\prime}$ ? In 1967, M. Tomita [25], [26] answered this question affirmatively by a new and original analysis of the spatial relationship between a von Neumann algebra and its commutant. The exposition of [25] and [26] was somewhat obscure, however, and in 1970, M. Takesaki published his seminal monograph [6] which explained and extended Tomita's earlier work. Let $M$ be a von Neumann algebra with a vector which is both cyclic and separating for M. Takesaki associated a closed, densely defined, self-adjoint operator $\Delta$ with $M$, the modular operator, which has two very useful properties. The first is that $\left\{\Delta^{i t}: t \in(-\infty, \infty)\right\}$ forms a one-parameter unitary group for which $\Delta^{-i t} M \Delta^{i t}=M$, the modular automorphism group of $M$, and the second is that $\Delta$ induces a conjugate-1inear, involutive isometry $\mathrm{J}$ of the underlying Hilbert space for which $\mathrm{JMJ}=\mathrm{M}^{\prime}$. This shows in particular that $M$ and $M^{\prime}$ are anti-isomorphic, and is the key to Tomita's proof of the commutation theorem for tensor products. In actuality, the existence of a cyclic and separating vector is not necessary for the definition 
of the modular operator, and in fact if $\varphi$ is any faithful, normal, positive linear functional on $M$, then the modular operator $\Delta_{\varphi}$ and the modular automorphism group $\left\{\Delta_{\varphi}^{i t}\right\}$ corresponding to $\varphi$ can be constructed in $H_{\varphi}$ relative to $\pi_{\varphi}(M)$, where $\left\{\pi_{\varphi}, \mathrm{H}_{\varphi}\right\}$ is the representation of $M$ arising from the GNS construction induced by $\varphi$. The construction of the modular operator and the verification of its main properties are quite technical, and for that reason we will not go into the details. We instead refer the reader to Rieffel and van Daele's excellent development of the Tomita-Takesaki theory [27], and, of course, also to the original memoir [6].

The stage was now set, and in 1973 Connes' thesis [5] appeared. This work contained a classification scheme for factors of type III which was to have a profound influence on all subsequent work in this area. Taking his cue from the Araki-Woods classification scheme and the Tomita-Takesaki theory, Connes introduced the modular spectrum as an isomorphism invariant for type III von Neumann algebras. Let $M$ be a separable acting type III von Neumann algebra. The modular spectrum $S(M)$ of $M$ is the intersection of the Arveson spectra (see [29]) of the modular operators $\Delta_{\varphi}$ corresponding to all faithful, normal, positive linear functionals $\varphi$ on $M$. Connes and van Daele proved the remarkable fact that $S(M) \backslash\{0\}$ forms a subgroup of $(0, \infty)$ and that $S(M)$ is a closed set which is an isomorphism invariant of $\mathrm{M}$. Connes then divided the type III von Neumann algebras into subtypes as follows: $M$ is said to be

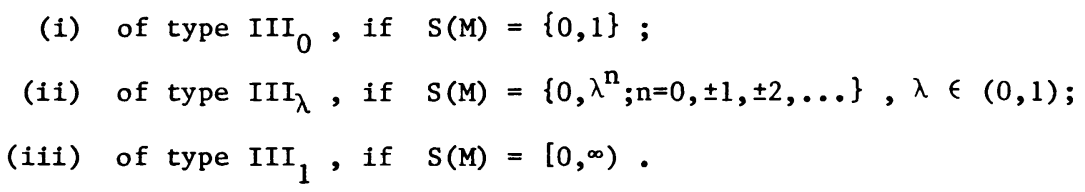

This is a direct generalization of the Araki-Woods classification: for a type III ITPFI factor $M, r_{\infty}(M)=S(M)$, and so

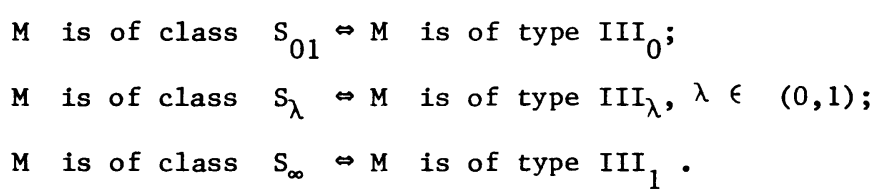


Connes then proceeded to prove a fundamental structure theorem for the factors of type $\operatorname{III}_{\lambda}, \quad \lambda \in[0,1)$ in terms of discrete crossed products of von Neumann algebras. The case of type III 1 was not treated, but Connes was not alone in this work. In the same year as Connes' thesis appeared, Takesaki also offered [28], which solved the III $_{1}$ case by the introduction of continuous crossed products, and which also developed a duality theory for crossed products that was to be very influential. We now proceed to describe the structure theorems of Connes and Takesaki.

Let $G$ be a locally compact abelian group, $M$ a von Neumann algebra. A continuous action $a$ of $G$ on $M$ is a homomorphism $\alpha$ of $G$ into Aut(M), the group of all *-automorphisms of $M$, such that for each $x \in M$, the mapping $g \rightarrow a_{g}(x)$ is *-strongly continuous. If $G=R$ (as will be the case in the sequel), we call a a one-parameter action on $M$.

Suppose $M$ acts on a Hilbert space $H$. Let $\lambda$ denote Haar measure on $G$, and let $L^{2}(G ; H)$ denote the Hilbert space of all $H$ - valued, $\lambda$-square integrable functions on $G$. We define the representations $\pi_{a}$ of $M$ and $\lambda$ of $G$ on $L^{2}(G ; H)$ as follows :

$$
\begin{aligned}
& \left(\pi_{\alpha}(x) \xi\right)(h)=a_{h}^{-1}(x) \xi(h) ; x \in M, h \in G, \xi \in L^{2}(G ; H) ; \\
& (\lambda(g) \xi)(h)=\xi\left(g^{-1} h\right) ; g, h \in G, \xi \in L^{2}(G ; H) .
\end{aligned}
$$

The crossed product $W^{*}(M, \alpha)$ of $M$ by $\alpha$ is the von Neumann subalgebra of $B\left(L^{2}(G ; H)\right)$ generated by $\left\{\pi_{\alpha}(x): x \in M\right\}$ and $\{\lambda(g): g \in G\}$. If $\theta \in \operatorname{Aut}(M)$, the crossed product $W^{*}(M, \theta)$ of $M$ by $\theta$ is the crossed product of $M$ by the discrete action $n \rightarrow \theta^{n}, n=0, \pm 1, \pm 2, \ldots$ We will of ten refer to $W^{*}(M, \theta)$ as a discrete crossed product or a discrete decomposition.

The oldest, and in many ways the most important, example of a crossed product is the classical group-measure space construction of Murray and von Neumann. Let $(\mathrm{X}, \mu)$ be a $\sigma-$ finite measure space with positive measure $\mu$, and call a bijective mapping $T$ of $X$ onto $X$ an automorphism of $X$ if $T$ and $T^{-1}$ are 
measurable and $T$ maps sets of $\mu$-measure zero to sets of $\mu$ - measure zero. An automorphism $T$ of $X$ induces an algebra automorphism $a_{T}$ of $L^{\infty}(X, \mu)$ in the natura1 way:

$$
\alpha_{\mathrm{T}}: \mathrm{f} \rightarrow \mathrm{f} \circ \mathrm{T} ; \mathrm{f} \in \mathrm{L}^{\infty}(\mathrm{X}, \mu)
$$

Let $H=L^{2}(X, \mu)$ denote the Hilbert space of all $\mu$-square integrable functions on $X L^{\infty}(X, \mu)$ acts by pointwise multiplication on $H$ and thereby forms a maximal abelian von Neumann subalgebra of $B(H)$, and $\alpha_{T}$ is a *-automorphism of this von Neumann algebra. The von Neumann algebra given by the group-measure space construction is simply the discrete crossed product $W^{*}\left(L^{\infty}(X, \mu), \alpha_{T}\right)$. $W *\left(L^{\infty}(X, \mu), \alpha_{T}\right)$ is hyperfinite, and if $T$ is ergodic, it is a factor. Two automorphisms $T_{1}$ and $T_{2}$ of $X$ are weakly equivalent if there exists an automorphism $U$ of $X$ such that $\left\{T_{1}^{n}(x): n \in Z\right\}=\left\{\left(U_{2}{ }^{n_{U}}{ }^{-1}\right)(x): n \in Z\right\}$ for $\mu$ - allmost all $\mathrm{x}$. In a famous paper which generalized the work of Araki and Woods, Krieger [30] proved that if $\mathrm{T}_{1}$ and $\mathrm{T}_{2}$ are ergodic automorphisms of $X$ then $W^{*}\left(L^{\infty}(X, \mu), a_{T_{1}}\right)$ is isomorphic to $W^{*}\left(L^{\infty}(X, \mu), a_{T_{2}}\right)$ if and only if $\mathrm{T}_{1}$ and $\mathrm{T}_{2}$ are weakly equivalent. For this reason, Connes calls a discrete crossed product of an abelian von Neumann algebra by an ergodic automorphism of the algebra a Krieger $\underline{\text { factor, }}$ and so therefore will we. (Incidentally, Connes cites the work of Krieger (along with Araki-Woods [24]) in the introduction to [5] as being one of the primary motivations for developing his classification scheme for type III factors).

We are now in a position to state what may appropriately be called the first, second, and third fundamental structure theorems for type III factors.

2.2. THEOREM. (Takesaki, [28]). Let $M$ be a factor of type III. There exists a von Neumann algebra $N$ of type $\mathrm{II}_{\infty}$, a semifinite, faithful, normal trace $\tau$ on $N$, and a one-parameter action $\alpha=a_{t}$ on $N$ such that

(i) $\tau \circ a_{t}=e^{-t} \tau$;

(ii) $M$ is isomorphic to $W *(N, \alpha)$. 
2.3. THEOREM. (Connes, [5]). Let $M$ be a factor of type $\operatorname{III}_{\lambda}, \lambda \in(0,1)$. There exists a factor $\mathrm{N}$ of type $\mathrm{II}_{\infty}$, an automorphism $\theta$ of $\mathrm{N}$, and a faithful normal, semifinite trace $\tau$ of $N$ such that

(i) $\tau \circ \theta=\lambda \tau$;

(ii) $M$ is isomorphic to $W^{*}(\mathrm{~N}, \theta)$.

Furthermore, any pair $(\mathrm{N}, \theta)$ satisfying (i) gives rise to a crossed product factor of type III $\lambda_{\lambda}$, and any two such pairs $\left(\mathrm{N}_{1}, \theta_{1}\right),\left(\mathrm{N}_{2}, \theta_{2}\right)$ give isomorphic factors if and only if they correspond to the same $\lambda$ and there is an isomorphism $\pi$ of $\mathrm{N}_{1}$ onto $\mathrm{N}_{2}$ such that $\mathrm{p}\left(\pi \theta_{1} \pi^{-1}\right)=\mathrm{p}\left(\theta_{2}\right)$, where $\mathrm{p}$ is the canonical quotient map of $\operatorname{Aut}\left(\mathrm{N}_{2}\right)$ onto the quotient of $\operatorname{Aut}\left(\mathrm{N}_{2}\right)$ by its subgroup of inner automorphisms.

2.4. THEOREM. (Connes, [5]). Let $M$ be a factor of type III $_{0}$. There exists a von Neumann algebra $N$ of type $I_{\infty}$ with a diffuse center, an automorphism $\theta$ of $N$ which is ergodic on the center of $N$, and a faithful, normal, semifinite trace $\tau$ on $\mathbf{N}$ such that

(i) for some $\lambda_{0}<1, \tau(\theta(x)) \leq \lambda_{0} \tau(x)$, for all positive elements $x$ of $\mathrm{N}$;

(ii) $M$ is isomorphic to $W^{*}(N, \theta)$. Any pair $(N, \theta)$ satisfying the above conditions gives rise to a factor of type III $0^{\cdot}$

These theorems in principle reduce the study of factors of type III to the study of von Neumann algebras of type $\mathrm{II}_{\infty}$ and their automorphisms, and Connes lost no time in beginning such a study.

If $M$ is a II $_{\infty}$ factor acting on a separable Hilbert space $H$, a theorem of Murray and von Neumann ( [4], Theorem IX) allows one to write $M$ as the tensor product of a II $I_{1}$ factor $N$ and $B(H)$. Thus the study of automorphisms of $I I_{\infty}$ factors can be effectively reduced to the study of automorphisms of $I_{1}$ factors. Now the hyperfinite $\mathrm{II}_{1}$ factor is in many ways the simplest of all the $\mathrm{II}_{1}$ factors, and hence any program which aspires to a classification of automorphisms of II ${ }_{1}$ factors must first of all handle the case of the hyperfinite II 
factor $R$. In [9], this is precisely what Connes did. In this deep and penetrating study, he determined all outer conjugacy classes of automorphisms of $R$, and in particular showed that the quotient of Aut(R) by its subgroup of inner automorphisms is a simple group with only countably many conjugacy classes. By our previous comments, all automorphisms of the $\mathrm{II}_{\infty}$ factor $R_{0,1}=\mathrm{B}(\mathrm{H})$ can now be determined.

The factor $R_{0,1}$ is also of interest for another reason. An old question of Murray-von Neumann asked whether all hyperfinite II $_{\infty}$ factors are isomorphic to $\mathrm{R}_{0,1}$ ? This question arose naturally from their work on the hyperfinite II factor, and for a long time was viewed as one of the most important open questions in the theory of von Neumann algebras. In [10] it received an affirmative answer. The key to this answer lies in the concept of injectivity, an idea introduced by Tomiyama in [31] and exploited by him and others in the study of tensor products of von Neumann algebras (the terminology is due to Effros and Lance [14]). The great achievement of [10] was to identify injectivity and hyperfiniteness for separably acting factors, first in the $I_{1}$ case, then for the $I_{\infty}$ case, and finally for the type III case using the fundamental structure Theorems 2.2, 2.3, and 2.4. It is to a detailed discussion of these ideas that we now turn.

A few words are in order concerning the organization of the remainder of the paper. The main theorem occurs in Section 8, and asserts that all injective II 1 factors are isomorphic. In Section 3, we define injectivity for von Neumann algebras and give a tracial characterization of injectivity for finite factors. Sections 4 and 5 are concerned with establishing a certain type of finite dimensional approximation property for standardly acting, injective, finite factors which plays a central role in the proof of the main theorem. Section 6 discusses semidiscreteness for injective finite factors. Automorphisms of factors are briefly treated in Section 7, and several important results of Connes on automorphisms of II $_{1}$ factors are stated for use in the proof of the main theorem. Section 8 commences with the proof of the main theorem, Section 9 provides some necessary lemmas on embeddings in ultraproducts, and proof of the main theorem is completed 
in Section 10. The eleventh and final section uses the results of Sections 2 and 8, together with some results from [7] and [3], to obtain the classification of injective factors of type $\operatorname{II}_{\infty}$ and $\operatorname{III}_{\lambda}, \lambda \in[0,1)$.

3. INJECTIVE VON NEUMANN ALGEBRAS AND THE HYPERTRACE.

A von Neumann algebra $\mathrm{N}$ acting on a Hilbert space $\mathrm{H}$ is said to be $\underline{\text { in- }}$ jective if there is a projection $E$ of $B(H)$ onto $N$ with $\|E\|=1$. It follows easily that $E \geq 0$, and by a well-known theorem of Tomiyama ([31]), E is a conditional expectation, i.e.,

$$
E(a x b)=a E(x) b, \quad a, b \in N, \quad x \in B(H)
$$

Suppose now that $\mathrm{N}$ is an injective finite factor acting on $\mathrm{H}$. Let $\tau$ denote a tracial state on $\mathrm{N}$, and set $\psi=\tau \circ \mathrm{E}$, where $\mathrm{E}$ is a projection of norm 1 of $\mathrm{B}(\mathrm{H})$ onto $\mathrm{N}$. Then $\psi$ is a state on $\mathrm{B}(\mathrm{H})$ such that for all $\mathbf{x} \in \mathbf{B}(\mathrm{H}), \quad \mathbf{a} \in \mathrm{N}$,

$$
\begin{aligned}
\psi(x a) & =\tau(E(x a))=\tau(E(x) a) \\
& =\tau(a E(x))=\tau(E(a x)) \\
& =\psi(a x) .
\end{aligned}
$$

A state $\psi$ of $B(H)$ satisfying $(3.1)$ is called a hypertrace of $N$.

Conversely, suppose $\mathrm{N}$ is a finite factor acting standardly on $\mathrm{H}$ with hypertrace $\psi$. We will eventually show that this forces $N$ to be injective, but before doing this we need to recall some facts about the Hilbert space $L^{2}(N, \tau)$ of Segal ([32]). An element $x$ of $L^{2}(N, \tau)$ is a closed, densely defined operator on $\mathrm{H}$ affiliated with $\mathrm{N}$ in the sense of Definition 2.1 of [32]. A positive operator $\mathrm{T}$ affiliated with $\mathrm{N}$ is called integrable when its spectral measure $\mu(E)=\tau\left(\chi_{E}(T)\right)(E$ a Borel subset of $(0,+\infty))$ satisfies $\int_{0}^{\infty} \lambda \mathrm{d} \mu(\lambda)<\infty$, and we set $\tau(\mathrm{T})=\int_{0}^{\infty} \lambda \mathrm{d} \mu(\lambda) \cdot L^{2}(\mathrm{~N}, \tau)$ consists of all integrable $\mathrm{x}$ satisfying $\tau\left(x^{*} x\right)<\infty$, and the norm of $L^{2}(N, \tau)$ is given by $\|x\|=\tau\left(x^{*} x\right)^{\frac{1}{2}}$. N is contained in $L^{2}(N, \tau)$ as a dense submanifold, and for $a \in N,\|a\|=\tau(a * a)^{\frac{1}{2}}$ $=\|\mathrm{a}\|_{2}$.

If we restrict the hypertrace $\psi$ to $\mathrm{N}$, a tracial state on $\mathrm{N}$ results and so $\left.\psi\right|_{N}=\tau$. The Schwartz inequality for positive linear functionals therefore 
yields for each $A \in B(H)$,

$$
\begin{aligned}
& |\psi(a A)| \leq \psi(A * A)^{\frac{1}{2}} \psi(a * a)^{\frac{1}{2}}=\psi(A * A)^{\frac{1}{2}} \tau\left(a *_{a}\right)^{\frac{1}{2}} \\
& \leq \| \text { A } \| \tau\left(a *_{a}\right)^{\frac{1}{2}}, \forall a \in N .
\end{aligned}
$$

Thus each $A \in B(H)$ determines a bounded linear functional $\phi_{A}: a \rightarrow \psi(a A)$ on $L^{2}(N, \tau)$, and hence a unique $E(A)$ in $L^{2}(N, \tau)$ such that

$$
\tau(\mathrm{aE}(\mathrm{A}))=(\mathrm{a}, \mathrm{E}(\mathrm{A}) *) L^{2}(\mathrm{~N}, \tau)=\psi(\mathrm{aA}) \quad, \forall \mathrm{a} \in \mathrm{N} \text {. }
$$

Claim: If $A \geq 0$, then $\phi_{A}$ is positive on $N$. Let $a \in N, T \in B(H)$. Then

$$
\begin{aligned}
\psi(\mathrm{a} * \mathrm{aT} * \mathrm{~T}) & =\psi(\mathrm{aT} * \mathrm{Ta} *)(\psi \text { is a hypertrace }) \\
& =\psi(\mathrm{aT} *(\mathrm{aT} *)) \geq 0 .
\end{aligned}
$$

This verifies the claim.

We can now show that $E(A) \in N$ for $A \geq 0$, Since $E(A)$ is affiliated with $N$, it suffices by the double commutant theorem to show that $E(A)$ extends to a bounded operator on $\mathrm{H}=\mathrm{L}^{2}(\mathrm{~N}, \tau)$.

$$
\begin{aligned}
& \text { Let } a, b \in N,\|a\|_{2},\|b\|_{2} \leq 1 \text {. Then } \\
& \left|(E(A) a, b){ }_{H}\right|=|\tau(b * E(A) a)| \\
& =|\tau(a b * E(A))| \text {, by [32], Corollary } 11.2 \\
& =|\psi(a b * A)|=\left|\phi_{A}(a b *)\right| \text {, by (3.3) } \\
& \leq \phi_{\mathrm{A}}(\mathrm{a} * \mathrm{a})^{\frac{1}{2}} \phi_{\mathrm{A}}(\mathrm{bb} *)^{\frac{1}{2}} \text {, since } \phi_{\mathrm{A}} \geq 0 \\
& \leq\|\mathrm{A}\| \tau\left(\mathrm{a} \mathrm{a}^{*}\right)^{\frac{1}{4}} \tau\left(\mathrm{b} \mathrm{b}^{*}\right)^{\frac{1}{4}} \text {, by (3.2) } \\
& =\|\mathrm{A}\| \quad\|\mathrm{a}\|_{2}^{\frac{1}{2}}\|\mathrm{~b}\|_{2}^{\frac{1}{2}} \\
& \leq\|\mathrm{A}\| \text {. }
\end{aligned}
$$

Since $N$ is dense in $H=L^{2}(N, \tau)$, it follows that $E(A)$ extends to a bounded operator on $\mathrm{H}$ with $\|\mathrm{E}(\mathrm{A})\| \leq\|\mathrm{A}\|$.

We conclude that $E(A) \in N$, for all $A \in B(H)$. It is now straightforward to deduce from (3.3) that $\mathrm{A} \rightarrow \mathrm{E}(\mathrm{A})$ is a projection of norm 1 of $\mathrm{B}(\mathrm{H})$ onto $\mathrm{N}$. The following proposition now obtains:

3.1. PROPOSITION. Let $\mathrm{N}$ be a finite factor. $\mathrm{N}$ is injective if and only if $\mathrm{N}$ admits a hypertrace in its standard representation. 4. THE METHOD OF DAY FOR INJECTIVE FINITE FACTORS。

Let $\mathrm{N}$ be a von Neumann algebra, $\varphi$ a linear functional on $\mathrm{N}, \mathrm{u}$ a unitary 
in $\mathrm{N}$. We denote by $\varphi \circ$ ad $\mathrm{u}$ the linear functional

$$
x \rightarrow \varphi\left(u x u^{*}\right), x \in N
$$

4.1. PROPOSITION. Let $\mathrm{N}$ be an injective finite factor acting standardly in $\mathrm{H}$ - Then for each finite set $\left\{\mathrm{u}_{1}, \ldots, \mathrm{u}_{\mathrm{n}}\right\}$ of unitaries in $\mathrm{N}$ and $\varepsilon>0$, there exists a normal state $\varphi$ on $B(H)$ such that

$$
\left\|\varphi-\left(\varphi \circ \operatorname{adu}_{j}\right)\right\|<\varepsilon, j=1, \ldots, \mathrm{n}
$$

PROOF. The proof uses a separation argument first employed by Day [33] in the context of countable amenable discrete groups.

We consider the Banach space $\left(B(\mathrm{H})_{\star}\right)^{n}$ formed by taking the Banach space direct sum of $n$ copies of the predual of $B(H)$. Consider the set

$$
C=\left\{\left(\varphi-\left(\varphi \circ \text { ad } u_{1}\right), \ldots, \varphi-\left(\varphi \circ \text { ad } u_{n}\right)\right): \varphi \in B(H)_{*}\right\} \cdot
$$

To verify (4.1), it suffices to show that $0 \in$ norm closure of $\mathrm{C}^{-} \mathrm{C}^{-}$.

Suppose $0 \& \mathrm{C}^{-}$. Then there is a norm-continuous linear functional $\mathrm{f}$ on $\left(\mathrm{B}(\mathrm{H})_{\star}\right)^{\mathrm{n}}$ and $a>0$ such that

$\operatorname{Re} f\left(\varphi-\left(\varphi \circ\right.\right.$ ad $\left.u_{1}\right), \ldots, \varphi-\left(\varphi \circ\right.$ ad $\left.\left.u_{n}\right)\right) \geq \alpha, \forall \varphi \in B(H)_{*} \cdot$ Now there exist $x_{1}, \ldots, x_{n} \in B(H)$ such that $f(g)=\sum_{1} g_{i}\left(x_{i}\right)$ for $g=\left(g_{1}, \ldots, g_{n}\right)$ $\epsilon\left(\mathrm{B}(\mathrm{H})_{*}\right)^{\mathrm{n}} \cdot$ Hence

$$
\operatorname{Re} \sum_{j=1}^{n}\left(\varphi\left(x_{j}\right)-\varphi\left(u_{j} x_{j} u_{j}{ }^{*}\right)\right) \geq a, \forall \varphi \in B(H){ }_{*} \cdot
$$

By Proposition 3.1, N has a hypertrace $\psi$. By the bipolar theorem ([34, Theorem 2.14]), $\psi$ is the $\sigma(B(H) *, B(H))$ - limit of a net $\left(\varphi_{\alpha}\right)_{\alpha \in A}$ of normal states on $B(H)$. Since (4.2) holds for each $\varphi_{\alpha}$, we hence conclude that (4.2) holds for $\psi$. But $\psi\left(x_{j}\right)=\psi\left(u_{j} x_{j} u_{j}^{*}\right), j=1, \ldots n$. We conclude that $0 \in c^{-}$.

5. AN ANALOG OF FØLNER'S CONDITION FOR INJECTIVE FACTORS.

As mentioned in the previous section, Day used the technique of Proposition 4.1 to show that when $G$ is a countable, amenable, discrete group, there exists 
a sequence $\left\{\mathrm{F}_{\mathrm{n}}\right\}$ of normalized positive functions in $\ell^{1}(G)$ such that $\left\|g F_{n}-F_{n}\right\|_{1} \rightarrow 0$, for all $g \in G$. Somewhat earlier Følner [35] had given a stronger result by finding a sequence $\left\{S_{n}\right\}$ of finite subsets of $G$ such that $s_{n} \neq \emptyset$, for all $n$, and

$$
\frac{\left(\mathrm{gS}_{\mathrm{n}} \Delta \mathrm{S}_{\mathrm{n}}\right)^{\#}}{\mathrm{~S}_{\mathrm{n}}^{\#}} \rightarrow 0, \forall g \in G,
$$

where $A^{\#}=$ cardinality of $A \subseteq G$ and $\Delta$ denotes symetric difference.

Let $\mathrm{N}$ be an injective finite factor acting standardly in $\mathrm{H}$. The next proposition gives an analog of Fфlner's result for $\mathrm{N}$, and will play a crucial role in the seque1. Before giving its statement and proof, we need a rather technical lemma concerning certain approximations for positive Hilbert-Schmidt operators.

For its proof, consult [10], Theorem 1.22 or [37], Section 2.5 .

LEMMA. Let $\mathrm{H}$ be a Hilbert space. Let $\chi_{a}$ denote the characteristic function of the interval $(a,+\infty), a \geq 0$. Let $h_{1}, \ldots, h_{n}$ be positive HilbertSchmidt operators on $H$ such that

$$
\left\|h_{j}-h_{1}\right\|_{H S}<\varepsilon\left\|h_{1}\right\|_{H S}, j=1, \ldots, n,
$$

for some $\varepsilon>0$, where \|\|$_{H S}$ denotes the Hilbert-Schmidt norm. Then there exists $a>0$ such that $\chi_{a}\left(h_{1}\right) \neq 0$ and

$$
\sum_{j=1}^{n}\left\|x_{a}\left(h_{j}\right)-\chi_{a}\left(h_{1}\right)\right\|_{H S}^{2}<3 n \varepsilon\left\|x_{a}\left(h_{1}\right)\right\|_{H S}^{2} \text {. }
$$

5.1. PROPOSITION. Let $\mathrm{N}$ be an injective finite factor acting standardly in $H$. For each finite subset $\left\{x_{1}, \ldots, x_{n}\right\} \subseteq N$ and $\varepsilon>0$, there exists a finite-rank projection $E \in B(H)$ such that $E \neq 0$ and

$$
\left\|\left[E, x_{j}\right]\right\|_{H S}<\varepsilon\|E\|_{H S}, j=1, \ldots, n \text {. }
$$

(For operators $A$ and $B$, we denote $A B-B A$ by $[A, B]$. ) 
PROOF. By Proposition 4.1, for any set $\left\{u_{1}, \ldots, u_{n}\right\}$ of unitaries of $N$, we can find a normal state $\varphi$ of $B(H)$ such that

$$
\| \varphi-\left(\varphi \circ \text { ad } u_{j}\right) \|<\varepsilon, j=1, \ldots, n \text {. }
$$

Let $\rho$ denote the density matrix of $\varphi$, i.e.,

$$
\varphi(\bullet)=\operatorname{Tr}\left(\rho^{\bullet}\right), \rho \geq 0, \operatorname{Tr}(\rho)=1,
$$

where $\operatorname{Tr}=$ canonical trace on $B(H)$. For $x \in B(H)$, we have

$$
\varphi \circ \operatorname{ad~} u_{j}(x)=\varphi\left(u_{j} x u_{j}^{*}\right)=\operatorname{Tr}\left(\rho u_{j} x u_{j}{ }^{*}\right)=\operatorname{Tr}\left(\left(u_{j}{ }^{*} \rho u_{j}\right) x\right) \text {. }
$$

Thus $\varphi \circ$ ad $u_{j}-\varphi$ has density matrix $u_{j}{ }^{*} \rho u_{j}-\rho$ and since the norm of a normal functional on $\mathrm{H}$ is the trace norm of its density matrix, we conclude from (5.2) that

$$
\left\|u_{j} * \rho u_{j}-\rho\right\|_{T r}<\varepsilon, j=1, \ldots, n \text {. }
$$

For $j=1, \ldots, n$, set $h_{j}=u_{j} \star h u_{j}$, where $h=\rho^{\frac{1}{2}}$ is a positive HilbertSchmidt operator. By (5.3) and the Powers-Stфrmer inequality [36],

$$
\begin{aligned}
\left\|h_{j}-h\right\|_{H S} & \leq\left\|h_{j}^{2}-h^{2}\right\|_{T r}=\left\|u_{j}{ }^{*} \rho u_{j}-\rho\right\|_{T r} \\
<\varepsilon, j=1, \ldots, n, & \\
\|h\|_{H S} & =\left\|h^{2}\right\|_{T r}^{2}=\|\rho\|_{T r}=1 .
\end{aligned}
$$

Thus by the lemma, there exists $a>0$ such that $\chi_{a}(h) \neq 0$ and

$$
\left\|x_{a}\left(h_{j}\right)-\chi_{a}(h)\right\|_{H S}<\left(3(n+1) \varepsilon^{\frac{1}{2}}\right)^{\frac{1}{2}}\left\|x_{a}(h)\right\|_{H S}, j=1, \ldots, n \text {. }
$$

Let $E=\chi_{a}(h)$. Then $\chi_{a}\left(h_{j}\right)=u_{j} * E u_{j}$ and $\operatorname{Tr} E<\infty$. Thus $E$ has finite rank, and by $(5.4)$

$$
\left\|\mathrm{E}-\mathrm{u}_{\mathrm{j}} * \mathrm{E} \mathrm{u}_{\mathrm{j}}\right\|_{\mathrm{HS}}<\left(3(\mathrm{n}+1) \varepsilon^{\frac{1}{2}}\right)^{\frac{1}{2}}\|\mathrm{E}\|_{\mathrm{HS}}, \mathrm{k}=1, \ldots, \mathrm{n} .
$$

Claim: $\left\|\left[E, u_{j}\right]\right\|_{H S}=\left\|E-u_{j} * E u_{j}\right\|_{H S}$. Let $\left\{e_{\alpha}\right\}$ be an orthonormal basis for $H$, and let $A_{j}=u_{j} *\left[E, u_{j}\right]$. Then

$$
\begin{aligned}
\left\|E-u_{j} * E u_{j}\right\|_{H S}^{2} & =\left\|A_{j}\right\|_{H S}^{2}=\sum_{\alpha}\left(A_{j} A_{j}{ }^{*} e_{\alpha}, e_{\alpha}\right)^{2} \\
& =\sum_{\alpha}\left(\left[E, u_{j}\right]\left[E, u_{j}\right] * u_{j} e_{\alpha}, u_{j} e_{\alpha}\right)^{2}
\end{aligned}
$$




$$
=\left\|\left[E, u_{j}\right]\right\|_{H S}^{2}, j=1, \ldots, n \text {. }
$$

(5.1) now follows by the claim, (5.5), the arbitrariness of $\varepsilon$, and the fact that $\mathrm{N}$ is the span of its unitary group.

6. SEMIDISCRETENESS AND PURIFICATION OF FACTOR STATES.

Let $A$ be a $C *-a l g e b r a$ acting on a Hilbert space $H$. Let $H^{c}$ denote the conjugate Hilbert space with $\xi \rightarrow \xi^{c}$ a conjugate-linear isometry of $H$ onto $H^{c}$. For $x \in B(H)$, denote by $x^{c}$ the element in $B\left(H^{c}\right)$ such that $x^{c} \xi^{c}=(x \xi)^{c}$, $\xi \in H$. Let $A^{c}=\left\{x^{c}: x \in A\right\} \cdot A^{c}$ is a $C^{*}$-subalgebra of $B\left(H^{c}\right)$.

Let $\varphi$ be a factor state on A . Assume for simplicity that in the GelfandNaimark-Segal construction $\left(\pi_{\varphi}, \mathrm{H}_{\varphi}, \xi_{\varphi}\right)$ corresponding to $\varphi, \xi_{\varphi}$ is separating for $\pi_{\varphi}(A) "$. Let $A \odot A^{c}$ denote the algebraic tensor product of $A$ and $A^{c}$, and define $\tilde{\varphi}: A \odot A^{c} \rightarrow C$ by

$$
\tilde{\varphi}\left(\mathrm{a} \otimes \mathrm{b}^{\mathrm{c}}\right)=\left(\pi_{\varphi}(\mathrm{a}) \xi_{\varphi}, \Delta_{\varphi}{ }^{\frac{1}{2} \pi_{\varphi}}(\mathrm{b}) \xi_{\varphi}\right)_{\mathrm{H}_{\varphi}}, \mathrm{a} \in A, \mathrm{~b}^{\mathrm{c}} \in \mathrm{A}^{\mathrm{c}}
$$

where $\Delta_{\varphi}$ is the modular operator corresponding to $\varphi$, the existence of which follows from Tomita-Takesaki theory. $\tilde{\varphi}$ always extends to a state on $A \otimes_{\max } A^{c}$; if $\tilde{\varphi}$ extends to a state on $A \underset{\min }{\otimes} A^{c}$, the spatial tensor product on $H^{\otimes} H^{c}$, we say that $\varphi$ admits a purification.

Let $\mathrm{N}$ be a factor acting on $\mathrm{H}$ - An early result of Murray and von Neumann [1] asserts that the homomorphism $\eta$ defined on $N \ominus N^{\prime}$ by $\eta: a \otimes a^{\prime} \rightarrow a a^{\prime}$ is an isomorphism. $\mathrm{N}$ is semidiscrete (in the sense of Effros and Lance, [14]) if this isomorphism extends to an isomorphism of $\mathrm{N} \otimes_{\min } \mathrm{N}^{\prime}$, i.e., if

$$
\left\|\sum_{j=1}^{n} a_{j} \otimes b_{j}\right\|_{H \otimes H}=\left\|\sum_{j=1}^{n} a_{j} b_{j}\right\|_{H}, \forall a_{1}, \ldots, a_{n} \in N,
$$

Connes characterizes semidiscreteness in terms of purification of states as follows ([37], Section 2.8):

6.1. PROPOSITION. Let $\mathrm{N}$ be a factor on $\mathrm{H}$. Then $\mathrm{N}$ is semidiscrete if and only if $\mathrm{N}$ has a faithful normal state which admits a purification. Now, let $\mathrm{N}$ be an injective finite factor, with $\tau$ denoting the canonical trace. $\tau$ is normal and faithful. We claim that $\tau$ admits a purification. To 
see this notice first that the map $S$ induced by the involution and the canonical trace vector 1 on $\pi_{\tau}(\mathrm{N}) 1$, viz.., $\mathrm{S}: \mathrm{a}(1) \rightarrow a *(1)$, is isometric, since

$$
\|\mathrm{a}(1)\|_{\mathrm{H}_{\tau}}^{2}=\tau(\mathrm{a} * \mathrm{a})=\tau\left(\mathrm{a} \mathrm{a}^{*}\right)=\|\mathrm{a} *(1)\|_{\mathrm{H}}^{2}
$$

Thus, $\Delta_{\tau}=(S * S)^{\frac{1}{2}}=I$, whence for all $a \otimes b^{c} \in N \Theta N^{c}$,

$$
\tilde{\tau}(a \otimes b c)=\left(\pi_{\tau}(a) 1_{\tau}, \pi_{\tau}(b) 1_{\tau}\right)_{H_{\tau}}=\tau(b * a) \text {. }
$$

But by Proposition 5.1 and ([37], Section 2.7), we have the following inequality:

$$
\begin{aligned}
\left|\tau\left(\sum_{j=1}^{n} b_{j} a_{j}\right)\right| & =\left|\tau\left(\sum_{j=1}^{n} a_{j} b_{j}^{*}\right)\right| \\
& \leq\left\|\sum_{j=1}^{n} a_{j} \otimes b_{j}{ }^{c}\right\|_{H \otimes H} c, \forall a_{j}, b_{j} \in N_{.}
\end{aligned}
$$

It follows that $\tilde{\tau}$ is bounded on $\mathrm{N} \Theta \mathrm{N}^{\mathrm{c}}$ relative to the spatial tensor product norm, and therefore has an extension to $\mathrm{N} \otimes_{\min } \mathrm{N}^{\mathrm{c}}$. We can now deduce from Proposition 6.1:

6.2. PROPOSITION. Injective finite factors are semidiscrete.

7. AUTOMORPHISMS OF FACTORS.

Let $M$ be a von Neumann algebra. We set

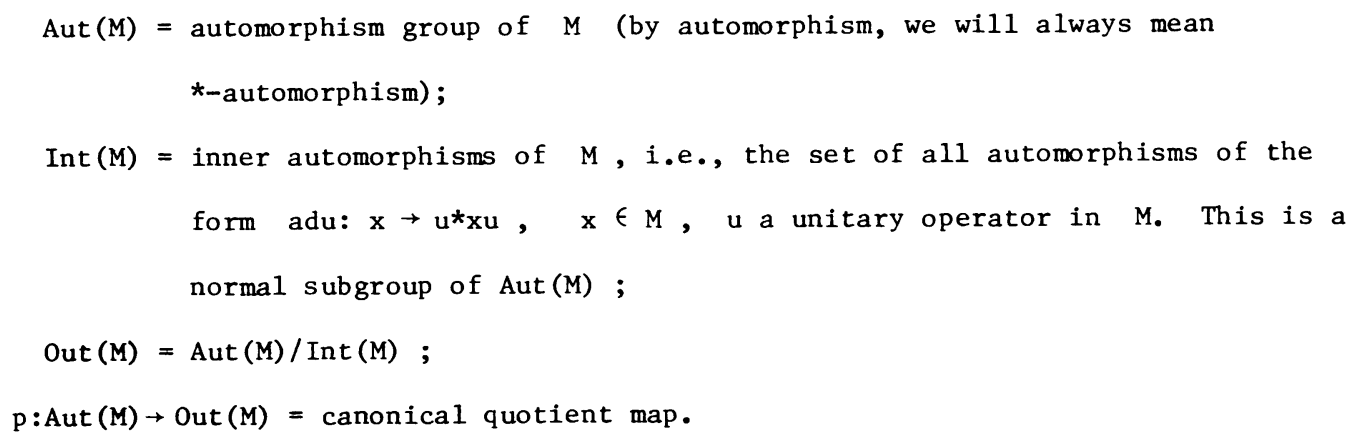

The weak topology on $A u t(M)$ is the topology of point-norm convergence in the predual $M_{\star}$ of $M$ for the action $\theta(\varphi)=\varphi\left(\theta^{-1}\right)$, i.e., a net $\left\{\theta_{a}\right\}$ of automorphisms converges weakly to $\theta \in$ Aut(M) if and only if $\lim _{\alpha}\left\|\varphi \circ \theta_{\alpha}^{-1}-\varphi \circ \theta^{-1}\right\|=0, \forall \varphi \in M_{*}$.

We set $\overline{\text { Int }} M=$ closure of Int $(M)$ in the topology just described.

Let $f$ be a fixed linear functional on $M$, and let a $\in M$. The linear 
functionals af and $f a$ on $M$ are defined respectively by af: $x \rightarrow f(x a)$, $\mathrm{fa}: \mathrm{x} \rightarrow \mathrm{f}(\mathrm{ax}), \mathrm{x} \in \mathrm{M}$. We set $[\mathrm{a}, \mathrm{f}]=\mathrm{af}-\mathrm{fa}$. A bounded sequence $\left\{\mathrm{x}_{\mathrm{n}}\right\} \subseteq \mathrm{M}$ is centralizing if $\left\|\left[x_{n}, \varphi\right]\right\| \rightarrow 0$, for all $\varphi \in M_{*}$. An automorphism $\theta$ of $M$ is centrally trivial if $\theta\left(x_{n}\right)-x_{n} \rightarrow 0, *-s t r o n g l y$, for tvery centralizing sequence $\left\{x_{n}\right\}$ of $M$ 。 We set

$C t(M)=$ group of all centrally trivial automorphisms of $M$. Ct(M) is a normal subgroup of Aut(M) .

7.1. LEMMA. $\operatorname{Int}(\mathrm{M}) \subseteq \operatorname{Ct}(\mathrm{M})$.

PROOF. We notice first that

(*) if $\left\{a_{n}\right\}$ is a bounded sequence in $M$ and $\left\|a_{n} \varphi\right\|,\left\|a_{n}{ }_{\varphi}\right\|$ both tend to 0 for all $\varphi \in M_{*}$, then $a_{n} \rightarrow 0 *$-strongly.

Let $\theta=$ ad $u \in \operatorname{Int}(M)$, and let $\left\{x_{n}\right\}$ be a centralizing sequence. We must show that $\theta\left(x_{n}\right)-x_{n} \rightarrow 0 *$-strongly. Now $\theta\left(x_{n}\right)-x_{n}=\left[u, x_{n}\right] u *$, and setting $a_{n}=\left[u, x_{n}\right] u *$, it suffices by $(*)$ to show that

$$
\underset{\mathrm{n}}{\lim }\left\|\left[\mathrm{u}, \mathrm{x}_{\mathrm{n}}\right] \varphi\right\|=\underset{\mathrm{n}}{\lim }\left\|\left[\mathrm{u}, \mathrm{x}_{\mathrm{n}}\right] * \varphi\right\|=0, \forall \varphi \in \mathrm{M}_{\star} \cdot
$$

But $\left[u, x_{n}\right] * \varphi=-\left[u *, x_{n}^{*}\right] \varphi$. Thus to verify (7.1), it suffices to show that for any unitary $u \in M$, any centralizing sequence $\left\{x_{n}\right\}$, and any $\varphi \in M_{*}$, we have

$$
\underset{\mathrm{n}}{\lim }\left\|\left[\mathrm{u}, \mathrm{x}_{\mathrm{n}}\right] \varphi\right\|=0
$$

Let $\psi \in M_{\star}$. One verifies by direct computation that for $y \in M$,

$$
\left[\mathrm{ux}_{\mathrm{n}} \mathrm{u}, \psi\right](\mathrm{y})=\left[\mathrm{x}_{\mathrm{n}}, \mathrm{u} * \psi \mathrm{u}\right](\mathrm{u} * \mathrm{yu}) .
$$

For $\varphi \in M_{*}, y \in M$.

$$
\begin{aligned}
{\left[u, x_{n}\right] \varphi(y) } & =\varphi\left(\operatorname{yux}_{n}-y x_{n} u\right)=u \varphi\left(y u x_{n} u^{*}-y x_{n}\right) \\
& =u \varphi\left(y u x_{n} u *-u x_{n} u * y\right)+u \varphi\left(u x_{n} u^{*} y-y x_{n}\right) \\
& =\left[u x_{n} u^{*}, u \varphi\right](y)+u \varphi\left(u x_{n} u^{*} y-y x_{n}\right) \\
& =\left[x_{n}, \varphi u\right](u * y u)+u \varphi\left(u x_{n} u^{*} y-y x_{n}\right), \text { by }(7.3) .
\end{aligned}
$$

Now,

$$
\begin{aligned}
u_{n} u * y-y x_{n} & =u x_{n} u^{*} y-y u * u x_{n} \\
& =\left(u x_{n} u^{*} y u^{*}-y u^{*} u x_{n} u^{*}\right) u
\end{aligned}
$$




$$
=\left[\mathrm{ux}_{\mathrm{n}} \mathrm{u}^{*}, \mathrm{yu}^{*}\right] \mathrm{u}
$$

Therefore,

$$
\begin{aligned}
u \varphi\left(u x_{n} u^{*} y-y x_{n}\right) & =u^{2} \varphi\left(\left[u x_{n} u^{*}, y u^{*}\right]\right) \\
& =-u^{2} \varphi\left(\left[y u^{*}, u x_{n} u^{*}\right]\right) \\
& =\left[u x_{n} u^{*},-u^{2} \varphi\right]\left(y u^{*}\right) \\
& =-\left[x_{n}, u \varphi u\right]\left(u^{*} y\right), \text { by }(7,3) .
\end{aligned}
$$

Thus by (7.4) and (7.5),

$$
\left[u, x_{n}\right] \varphi(y)=\left[x_{n}, \varphi u\right](u * y u)-\left[x_{n}, u \varphi u\right](u * y) .
$$

(7.2) now follows from (7.6) and the fact that $\left\{x_{n}\right\}$ is centralizing.

7.2. LEMMA. Let $M$ be a von Neumann algebra with separable predual. Then for each $\theta \in C t(M), a \in \overline{\text { Int } M}$,

$$
p(\theta) p(\alpha)=p(\alpha) p(\theta) \text {. }
$$

PROOF. For $\varphi \in M_{\star}$, denote the seminorm $x \rightarrow \varphi\left(x^{*} x\right)^{\frac{1}{2}}$ by \|\|$_{\varphi}$. Since $\theta$ is centrally trivial, for each positive integer $n$ there is a neighborhood $v_{n}$ of 1 in $\operatorname{Aut}(M)$ such that for all unitaries $u \in M$ with ad $u \in v_{n}$,

$$
\begin{aligned}
& \|\theta(u)-u\|_{\varphi \circ a^{-1}<2^{-n},} \\
& \|\theta(u)-u\|_{\varphi \circ\left(\theta a^{-1} \theta^{-1}\right)}<2^{-n} .
\end{aligned}
$$

Let $\left\{w_{n}\right\}$ be a decreasing basis of heighborhoods of $a$ in Aut(M) such that $\mathrm{w}_{\mathrm{n}} \mathrm{W}_{\mathrm{n}}^{-1} \subseteq \mathrm{v}_{\mathrm{n}}$ and $\left\|\varphi \circ \beta^{-1}-\varphi \circ \alpha^{-1}\right\|<2^{-2 \mathrm{n}}$. Let $u_{n}$ be a unitary in $M$ such that $\operatorname{ad} u_{n} \in w_{n}$. Then $\theta a \theta^{-1}=\lim _{n} \operatorname{ad} \theta\left(u_{n}\right)$, so we must show that $u_{n} * \theta\left(u_{n}\right)$ converges *-strongly to a unitary in $M$.

$$
\text { Let } v_{n}=u_{n+1} u_{n}^{*} \text {, so that } v_{n} \in w_{n} W_{n}^{-1} \text {. Then }
$$

$\left\|\theta\left(v_{n}\right)-v_{n}\right\|_{\varphi \circ a^{-1}<2^{-n}}$, and therefore

$$
\left\|\theta\left(v_{n}^{*}\right) v_{n}-1\right\| \operatorname{\varphi ddu}_{n}^{*} \quad<2 \cdot 2^{-n}+2^{-n}=3 \cdot 2^{-n},
$$


since ad $u_{n} \in W_{n}$. Thus

$$
\left\|\theta\left(v_{n}^{*}\right) v_{n} u_{n}-u_{n}\right\|_{\varphi}<3 \cdot 2^{-n}
$$

and

$$
\left\|\theta\left(u_{n+1}^{*}\right) u_{n+1}-\theta\left(u_{n}^{*}\right) u_{n}\right\|_{\varphi}=\left\|\theta\left(u_{n}^{*}\right) \theta\left(v_{n}^{*}\right) v_{n} u_{n}-\theta\left(u_{n}^{*}\right) u_{n}\right\|_{\varphi}<3 \cdot 2^{-n} \text {. }
$$

Also, because

$$
\left\|\theta\left(v_{n+1}\right)-v_{n+1}\right\|_{\varphi \circ\left(\theta a^{-1} \theta^{-1}\right)}<2^{-n},
$$

and

$$
\left\|\varphi \circ\left(\operatorname{ad} \theta\left(u_{n}^{-1}\right)\right)-\varphi \circ\left(\theta a^{-1} \theta^{-1}\right)\right\|<2^{-n},
$$

we obtain

$$
\left\|u_{n+1} * \theta\left(u_{n+1}\right)-u_{n} * \theta\left(u_{n}\right)\right\|_{\varphi}<3 \cdot 2^{-n}
$$

This shows that $u_{n} * \theta\left(u_{n}\right)$ converges *-strongly to a unitary $u \in M$ such that ad $u=a^{-1} \theta a \theta^{-1}$

The relation between Int $(M), \overline{\operatorname{Int}}(M)$, and $C t(M)$ has a strong bearing on the structure of $M$. By Lemma 7.2, one always has $p(\operatorname{Ct}(M)) \subseteq$ commutant of $p$ (Int $M$ )。 On the other hand, Connes proves the following remarkable theorem:

7.3. THEOREM ([9], Theorem 2.2.1). Let $M$ be a factor with separable predual, and let $R$ denote the hyperfinite factor of type $I_{1}$. The following are equivalent:

(a) $M$ is isomorphic to $M \otimes R$.

(b) $\mathrm{p}(\overline{\text { Int }} \mathrm{M})$ is nonabelian.

(c) $\overline{\operatorname{In} t} \mathrm{M} \notin \operatorname{Ct}(\mathrm{M})$.

Moreover, if (a) holds, then $\mathrm{p}(\mathrm{Ct}(\mathrm{M}))=$ commutant of $\mathrm{p}(\overline{\mathrm{Int}} \mathrm{M})$.

We now list several theorems which allow us to manipulate $\overline{\operatorname{Int}} \mathrm{N}$ and $\operatorname{Ct}(\mathrm{N})$ for a factor $\mathrm{N}$ of type $\mathrm{II}_{1}$ - Regretfully we must omit all proofs; for details, consult the indicated references (all results are due to Connes)。

Let $\mathrm{N}$ be a finite factor, $\tau$ the canonical trace on $\mathrm{N}$ 。 Recall that $\mathrm{N}$ has property $\Gamma$ of Murray and von Neumann ([4]) if for each finite subset 
$\left\{x_{1}, \ldots, x_{n}\right\}$ of $N$ and $\varepsilon>0$, there is a unitary $u \in M$ with $\tau(u)=0$ and $\left\|\left[u, x_{j}\right]\right\|_{2}<\varepsilon, j=1, \ldots, n$.

7.4. THEOREM. ([7], Corollary 3.8) . Let $\mathrm{N}$ be a II factor with separable predua1. Then

$\operatorname{Int}(\mathrm{N}) \neq \overline{\operatorname{Int}} \mathrm{N}$ if and only if $\mathrm{N}$ has property $\Gamma$.

7.5. THEOREM. ([10], Theorem 2.1) . Let $\mathrm{N}$ be a II factor acting standardly on $\mathrm{H}$. Let $K$ denote the compact operators on $\mathrm{H}, \mathrm{C} *\left(\mathrm{~N}, \mathrm{~N}^{\prime}\right)$ the $\mathrm{C}^{*}-$ algebra generated by $\mathrm{N}$ and $\mathrm{N}^{\prime}$. Then

$\mathrm{N}$ has property $\Gamma$ if and only if $\mathrm{C}^{*}\left(\mathrm{~N}, \mathrm{~N}^{\prime}\right) \cap K=(0)$.

Since $\mathrm{N}$ acts standardly on $\mathrm{H}, \mathrm{C} *\left(\mathrm{~N}, \mathrm{~N}^{\prime}\right)$ is irreducible. The next theorem gives a complete characterization of $\overline{\text { Int } N}$ for $I_{1}$ factors.

7.6. THEOREM ([10], Theorem 3.1) - Let $\mathrm{N}$ be a $\mathrm{II}_{1}$ factor acting standardly on $\mathrm{H}$. Then $\theta \in \overline{\text { Int }} \mathrm{N}$ if and only if $\theta$ extends to an automorphism $a \in \operatorname{Aut}\left(\mathrm{C} *\left(\mathrm{~N}, \mathrm{~N}^{\prime}\right)\right)$ such that

$$
\left.a\right|_{N}=\theta,\left.\quad a\right|_{N^{\prime}}=\text { Identity on } N^{\prime} \text {. }
$$

7.7. THEOREM. ([10], Corollary 4.4). Let $\mathrm{N}_{1}, \mathrm{~N}_{2}$ be II factors. Then for $\theta_{i} \in \operatorname{Aut}\left(\mathrm{N}_{i}\right), i=1,2$,

$\theta_{1} \otimes \theta_{2} \in \operatorname{Ct}\left(N_{1} \otimes N_{2}\right)$ if and only if $\theta_{i} \in \operatorname{Ct}\left(N_{i}\right)$, $i=1,2$.

8. UNIQUENESS OF THE INJECTIVE II $I_{1}$ FACTOR.

In this section, we begin the proof of the main theorem. The proof will end in Section 10.

8.1 THEOREM. ([10], Theorem 5.1) . All injective factors of type II acting on a separable Hilbert space are isomorphic.

PROOF. Let $\mathrm{N}$ denote an injective $\mathrm{II}_{1}$ factor acting on a separable Hilbert space, and acting standardly on $H=L^{2}(N, \tau)$. We will show that $N$ is isomorphic to the hyperfinite $\operatorname{II}_{1}$ factor $R$.

By Proposition $6.2, N$ is semidiscrete, $i_{\circ} e_{\circ}$, the mapping $\eta: N \circlearrowleft N^{\prime} \rightarrow B(H)$ given by $\eta: \sum_{1}^{n} a_{i} \otimes b_{i} \rightarrow \sum_{1}^{n} a_{i} b_{i}$ is isometric as a mapping from $B(H \otimes H) \rightarrow B(H)$ 。 
Since

$$
C *\left(N, N^{\prime}\right)=\text { norm closure of }\left\{\sum_{1}^{n} a_{i} b_{i}: a_{i} \in N, b_{i} \in N^{\prime}, n \in Z_{+}\right\} \text {, }
$$

it follows that $\eta$ extends to an isomorphism of $N \otimes N^{\prime}$ onto $C^{*}\left(N, N^{\prime}\right)$.

We claim that $N$ and $N^{\prime}$ are simple, i.e., they contain no closed, twosided ideals. Since $\mathrm{N}$ is a finite factor, by [38], Theorem $6.2, \mathrm{~N}$ contains no nonzero maximal ideals. Since every two-sided ideal of $\mathrm{N}$ is contained in a maximal one, we conclude that $\mathrm{N}$ is simple. Since $\mathrm{N}$ is conjugate-linearly isomorphic to $\mathrm{N}^{\prime}$ ([13], Proposition 2.9.2), it follows that $\mathrm{N}^{\prime}$ is also simple.

By a theorem of Takesaki ([39], we conclude that $N \otimes N^{\prime}$ is simple so that min

$\mathrm{C} *\left(\mathrm{~N}, \mathrm{~N}^{\prime}\right)$ is simple. Hence if $K$ denotes the (closed, two-sided) ideal of compact operators on $\mathrm{H}$, then $\mathrm{C}^{*}\left(\mathrm{~N}, \mathrm{~N}^{\prime}\right) \cup K$ is either $(0)$ or $\mathrm{C} *\left(\mathrm{~N}, \mathrm{~N}^{\prime}\right)$. Since $I \in C *\left(N, N^{\prime}\right)$ and $H$ is infinite-dimensional, $C *\left(N, N^{\prime}\right) \cap_{K} \neq C *\left(N, N^{\prime}\right)$, so $\mathrm{C} *\left(\mathrm{~N}, \mathrm{~N}^{\prime}\right) \cap K=(0)$. Thus by Theorems 7.4 and 7.5 ,

$$
\operatorname{Int}(\mathrm{N}) \neq \overline{\operatorname{In} t} \mathrm{~N} \text {. }
$$

Let $\sigma_{F}$ denote the flip automorphism of $N \otimes N$, i.e.,

$$
\sigma_{F}(x \otimes y)=y \otimes x, \quad x, y \in N .
$$

We want to show that $\sigma_{F} \in \overline{\operatorname{Int}}(\mathrm{N} \otimes \mathrm{N})$.

To do this, let $M$ be a standardly acting semidiscrete II $_{1}$ factor, and let $\theta \in \operatorname{Aut}(M)$. Then for $a 11 a_{1}, \ldots, a_{n} \in M, b_{1}, \ldots, b_{n} \in M^{\prime}$,

$$
\begin{aligned}
\left\|\sum_{1}^{n} a_{i} b_{i}\right\| & =\left\|\sum_{1}^{n} a_{i} \otimes b_{i}\right\| \quad(M \text { is semidiscrete) } \\
& =\left\|\sum_{1}^{n} \theta\left(a_{i}\right) \otimes b_{i}\right\| \begin{array}{l}
\text { since } \theta \otimes \text { id extends to } \\
\text { an automorphism of } M_{\text {min }} M^{\prime}
\end{array} \\
& =\left\|\sum_{1}^{n} \theta\left(a_{i}\right) b_{i}\right\| \quad \text { (semidiscreteness again) }
\end{aligned}
$$

This shows that $\theta$ extends to an automorphism $\alpha$ of $C *\left(M, M^{\prime}\right)$ such that $\left.a\right|_{M}=\theta,\left.\quad a\right|_{M^{\prime}}=$ identity on $M^{\prime}$ 。 Thus by Theorem $7.6, \theta \in \overline{\text { Int }} M$. We have hence shown that

$$
\text { Aut } M=\overline{\operatorname{In} t} M_{0}
$$


Now by a theorem of Effros and Lance ([14], Proposition 5.6) $N \otimes N$ inherits semidiscreteness from $N$, and standard facts about tensor products imply that $N \otimes N$ is a standardly acting $I_{1}$ factor. We hence conclude that Aut $(N \otimes N)=$ $\overline{\operatorname{Int}}(\mathrm{N} \otimes \mathrm{N})$, and so $\sigma_{\mathrm{F}} \in \overline{\operatorname{Int}}(\mathrm{N} \otimes \mathrm{N})$.

Since $\mathrm{N}$ acts on a separable Hilbert space, so does $\mathrm{N} \otimes \mathrm{N}$, and therefore $N \otimes N$ has separable predual. By Lemma 7.2, $p(C t(N \otimes N)) \subset$ commutant of $\mathrm{p} \overline{(\operatorname{Int}}(\mathrm{N} \otimes \mathrm{N}))$, whence by Theorem 7.7,

$$
p(\theta \otimes 1) p\left(\sigma_{F}\right)=p\left(\sigma_{F}\right) p(\theta \otimes 1), \forall \theta \in C t(N)
$$

Since $\sigma_{F}(\theta \otimes 1) \sigma_{F}^{-1}=1 \otimes \theta$, we get

$$
p(1 \otimes \theta)=p\left(\sigma_{F}(\theta \otimes 1) \sigma_{F}^{-1}\right)=p(\theta \otimes 1),
$$

whence

$$
\theta \otimes \theta^{-1} \in \operatorname{Int}(N \otimes N)
$$

Now a theorem of R. Kallman ([40], Corollary 1.14) asserts that if $M_{1}$ and $M_{2}$ are von Neumann algebras, $\theta_{i} \in \operatorname{Aut}\left(M_{i}\right), i=1,2$, then $\theta_{1} \otimes \theta_{2}$ $\operatorname{Int}\left(M_{1} \otimes M_{2}\right)$ if and only if either $\theta_{1} \notin \operatorname{Int}\left(M_{1}\right)$ or $\theta_{2} \| \operatorname{Int}\left(M_{2}\right)$. Thus by (8.2), $\theta \in \operatorname{Int}(\mathrm{N})$. Recalling Lemma 7.1, we have thus shown that

$$
\operatorname{Ct}(\mathrm{N})=\operatorname{Int}(\mathrm{N})
$$

We conclude from (8.1) and (8.3) that

$$
\operatorname{Ct}(\mathrm{N})=\operatorname{Int}(\mathrm{N}) \underset{\neq}{\subset} \overline{\operatorname{Int}} \mathrm{N},
$$

so by Theorem 7.3, $\mathrm{N}$ is isomorphic to $\mathrm{N} \otimes \mathrm{R}$.

This completes the first major step of the proof of Theorem 8.1 . In order to fully exploit this isomorphism, we must relate $N$ more closely to $R$ (after all, we are trying to show that $N$ and $R$ are in fact the same). This is done by embedding $\mathrm{N}$ in the ultraproduct $\mathrm{R}^{\omega}, \omega$ a free ultrafilter. We take up the details in the next section and it is there that Proposition 5.1 plays a crucial role.

9. EMBEDDINGS OF $\mathrm{N}$ IN ULTRAPRODUCTS 。 Let $z_{+}$denote the positive integers with the discrete topology. Let 
$C_{b}\left(Z_{+}\right)=\ell^{\infty}$ denote the $C^{*}$-algebra of bounded sequences. We identify the Stone-Čech compactification $\beta Z_{+}$of $Z_{+}$with $M_{\ell}^{\infty}$, trut maximal ideal space of $\ell^{\infty}$. Points $n \in Z_{+}$correspond to the homomorphism of evalutaion at $n$, and free ultrafilters $\omega \in \beta z_{+} \backslash z_{+}$correspond to nomomorphisms not of this form. Suppose $\omega \in \beta Z_{+} \backslash Z_{+}$corresponds to $\phi \in M_{l}^{\infty}$. Then for a sequence $\left\{a_{n}\right\} \in \ell^{\infty}$, we write $\lim _{n \rightarrow \infty} a_{n}=a$ if $\phi\left(\left\{a_{n}\right\}\right)=a$. Now, let $\left(\mathrm{N}_{\mathrm{k}}\right)_{\mathrm{k} \in \mathrm{Z}_{+}}$be a sequence of factors with finite normalized traces $\tau_{k}$, and let $\omega$ be a free ultrafilter on $z_{+} \cdot$ On $\oplus{ }_{1}^{\infty} N_{k}$, the C*-direct sum of the $\mathrm{N}_{\mathrm{k}}$ 's , we define the trace

$$
\tau_{\omega}:\left(x_{k}\right) \rightarrow \lim _{k \rightarrow \omega} \tau_{k}\left(x_{k}\right)
$$

Let $z_{\omega}=$ kernel of $\tau_{\omega}=\left\{x \in \oplus_{1}^{\infty} N_{k}: \tau_{\omega}\left(x^{*} x\right)=0\right\} . z_{\omega}$ is a closed, two-sided ideal in $\oplus_{1} \mathrm{~N}_{\mathrm{k}}$. Let

$$
\Pi_{\omega} \mathrm{N}_{k}=\oplus{ }_{1}^{\infty} \mathrm{N}_{k} / \mathrm{Z}_{\omega}
$$

Then ([41], p. 451) $\Pi_{\omega} \mathrm{N}_{k}$ is a von Neumann algebra with finite normalized trace $\tau_{\omega}$, called the ultraproduct of the $\mathrm{N}_{\mathrm{k}}$ 's corresponding to $\omega$.

Denote by $M^{\omega}$ the ultraproduct formed by countably many copies of the factor M. We proceed to construct an embedding of $\mathrm{N}$ into $\mathrm{R}^{\omega}$.

Let $F_{\mathrm{n}}$ denote the free group on the generators $\mathrm{g}_{1}, \ldots, \mathrm{g}_{\mathrm{n}}$. If $\mathrm{m} \in F_{\mathrm{n}}$, we define the length of $m$ as the sum of the absolute values of all exponents appearing in a reduced presentation of $\mathrm{m}$. Let $\mathrm{F}_{\mathrm{k}}=\operatorname{set}$ of $\mathrm{all} \mathrm{m} \in F_{\mathrm{n}}$ of length $\leq \mathrm{k}$.

Each $n-t u p l e ~ u=\left(u_{1}, \ldots, u_{n}\right)$ of unitaries defines a unitary representation $\mathrm{u}$ of $F_{\mathrm{n}}$ as follows: if $\mathrm{m} \in F_{\mathrm{n}}, \mathrm{u}(\mathrm{m})$ is the element formed by replacing each $g_{i}$ occuring in $m$ by $u_{i}, i=1, \ldots, n$.

9.1. LEMMA。 Let $u_{1}, \ldots, u_{n}$ be unitaries in $N$ 。 For each $\varepsilon>0$, there is a finite-dimensional factor $Q$ and unitary operators $v_{1}, \ldots, v_{n} \in Q$ such that 
$\left|\tau(\mathrm{u}(\mathrm{m}))-\tau_{q}(\mathrm{v}(\mathrm{m}))\right|<\varepsilon, \mathrm{m} \in \mathrm{F}_{\mathrm{k}}$, where $u=\left(u_{1}, \ldots, u_{n}\right), v=\left(v_{1}, \ldots, v_{n}\right)$, and $\tau_{q}$ is the normalized trace on Q •

PROOF. We first strengthen Proposition 5.1 as follows: given $\mathrm{x}_{1}, \ldots, \mathrm{x}_{\mathrm{n}} \in \mathrm{N}$ and $\varepsilon>0$, there exists a finite-rank projection $\mathrm{E} \neq 0$ on $\mathrm{H}$ such that for $j=1, \ldots, n$,

$$
\begin{aligned}
& \left\|\left[E, x_{j}\right]\right\|_{H S}<\varepsilon\|E\|_{H S}, \\
& \left|\tau\left(x_{j}\right)-\frac{\left(x_{j} E, E\right) H S}{\|E\|_{H S}^{2}}\right|<\varepsilon .
\end{aligned}
$$

To see this, we first claim that there exist unitaries $\left\{u_{1}, \ldots, u_{m}\right\} \subseteq N$ and $\delta>0$ such that for any state $\phi$ on $N$ with $\left\|\left[u_{j}, \phi\right]\right\| \leq \delta, j=1, \ldots, m$ one has $\left|\tau\left(x_{j}\right)-\phi\left(x_{j}\right)\right|<\varepsilon, j=1, \ldots, n$. For suppose not. Then for each finite subset $\sigma$ of unitaries of $N$ and $\delta>0$, there is a state $\phi_{\sigma, \delta}$ on $N$ such that

$$
\begin{aligned}
& \text { (i) }\left\|\left[u, \phi_{\sigma, \delta}\right]\right\| \leq \delta, \forall u \in \sigma, \\
& \text { (ii) }\left|\phi_{\sigma, \delta}\left(x_{j}\right)-\tau\left(x_{j}\right)\right| \geq \varepsilon \text {, for some } j \in\{1, \ldots, n\} \text {. }
\end{aligned}
$$

If we partially order the set of $(\sigma, \delta)$ 's by setting $\left(\sigma_{1}, \delta_{1}\right) \leq\left(\sigma_{2}, \delta_{2}\right)$ if $\sigma_{1} \subset \sigma_{2}$ and $\delta_{1} \leq \delta_{2}$, then $\left\{\phi_{\sigma, \delta}\right\}$ is a net and so by weak *-compactness of the state space of $N,\left\{\phi_{\sigma, \delta}\right\}$ weak $*$-accumulates at a state $\phi$ of $N$. By (i), $\phi$ is unitarily invariant, and so $\phi=\tau$. But since $\phi \sigma, \delta \rightarrow \phi=\tau$ (weak*), we may find $\phi_{\sigma, \delta}$ such that

$$
\left|\phi_{\sigma, \delta}\left(\mathrm{x}_{j}\right)-\tau\left(\mathrm{x}_{\mathrm{j}}\right)\right|<\varepsilon, j=1, \ldots, \mathrm{n},
$$

contradicting (ii). This verifies the claim.

Now use Proposition 5.1 to find a finite rank projection $E \neq 0$ such that for $i=1, \ldots, n, j=1, \ldots, m$, 
(iii) $\left\|\left[\mathrm{x}_{\mathrm{i}}, \quad \mathrm{E}\right]\right\|_{\mathrm{HS}}<\varepsilon\|\mathrm{E}\|_{\mathrm{HS}}$,

(iv) $\left\|\left[u_{j}, E\right]\right\|_{H S}<\frac{\delta}{2}\|E\|_{H S}$ 。

We assert that the state $\psi$ on $\mathrm{N}$ defined by

$$
\psi(x)=\frac{(x E, E)_{H S}}{\|E\|_{H S}^{2}}=\frac{\operatorname{Tr}(E x E)}{\operatorname{Tr} E}
$$

satisfies $\left\|\left[u_{j}, \psi\right]\right\| \leq \delta, j=1, \ldots, n$. This is so because

$$
\begin{aligned}
\psi_{u_{j}}(x)=\psi\left(u_{j} \times u_{j}^{*}\right) & =\frac{\operatorname{Tr}\left(E u_{j} x u_{j} * E\right)}{\operatorname{Tr} E} \\
& =\frac{\operatorname{Tr}\left(E u_{j} x u_{j} \star E u_{j} u_{j} *\right)}{\operatorname{Tr} E} \\
& =\frac{\operatorname{Tr}\left(\left(u_{j} \star E u_{j}\right) x\left(u_{j} \star E u_{j}\right)\right)}{\operatorname{Tr} E} \\
& =\frac{\left(x E_{j}, E E_{j}\right)_{H S}}{\operatorname{Tr} E}, E_{j}=u_{j} * E u_{j} .
\end{aligned}
$$

Therefore,

$$
\begin{aligned}
\mid \Psi(x) & -\psi_{u_{j}}(x)|=|(x E, E)_{H S}-\left(x E_{j}, E_{j}\right)_{H S} \mid \cdot\|E\|_{H S}^{-2} \\
& \leq\left(\left|\left(x\left(E-E_{j}\right), E\right)_{H S}\right|+\left|\left(x E_{j}, E-E_{j}\right)_{H S}\right| \cdot\|E\|_{H S}^{-2}\right. \\
& \leq\|x\|\left\|E-E_{j}\right\|_{H S}\|E\|_{H S}^{-2}\left(\|E\|_{H S}+\left\|E_{j}\right\|_{H S}\right) \\
& \leq\|x\| \frac{\delta}{2}\|E\|_{H S}^{-1} \cdot 2\|E\|_{H S}, \text { by (iv) } \\
& =\delta\|x\| .
\end{aligned}
$$

Since $\left[u_{j}, \psi\right](x)=\left(\psi-\psi_{u_{j}}\right)\left(x_{j}\right), x \in N$, we conclude that

$\left\|\left[u_{j}, \psi\right]\right\| \leq \delta, j=1, \ldots, n$, as asserted. Thus, by the previous claim,

$$
\left|\tau\left(x_{j}\right)-\frac{\left(x_{j} E, E\right)_{H S}}{\|E\|_{H S}^{2}}\right|<\varepsilon, j=1, \ldots, n,
$$


which together with (iii) is what we are seeking.

Now, letting $\delta=\varepsilon / 2 k$, we may hence find a nonzero finite-rank projection E such that

$$
\begin{aligned}
& \left\|\left[E, u_{j}\right]\right\|_{H S}<\delta\|E\|_{H S}, j=1, n, \\
& \mid \tau(u(m))-\frac{(u(m) E, E)}{\|E\|_{H S}^{2}} k \varepsilon, m \in F_{k} .
\end{aligned}
$$

Let $E_{j}=u_{j} E u_{j} *$; then $\left\|E{ }_{j}-E\right\|_{H S}<\delta\|E\|_{H S}$, and, by $[10]$, Lemma 1.4 , there are unitary operators $w_{j} \in B(H)$ with $w_{j} E_{j} w_{j}{ }^{*}=E$ and $\left\|w_{j}-I\right\|_{H S}<3 \delta\|E\|_{H S} \cdot$ Let $v_{j}^{\prime}=w_{j} u_{j} \cdot$ Then

$$
v_{j}{ }^{\prime} E=E v_{j}^{\prime},\left\|v_{j}{ }^{\prime}-u_{j}\right\|_{H S}<\frac{\varepsilon}{k}\|E\|_{H S}, j=1, \ldots, n .
$$

Let $v_{j}=v_{j}{ }^{\prime} E=E v_{j}^{\prime}$. Then $v_{j}$ is a unitary operator in the finite-dimensional factor $Q=E B(H) E$. The normalized trace $\tau_{q}$ on $Q$ is given by

$$
\tau_{q}(x)=\frac{(x E, E) H S}{\|E\|_{H S}^{2}}, \quad x \in Q
$$

Since for any unitaries $a, b \in B(H)$ we have

$$
\left\|a u_{j} b-a v_{j}{ }^{\prime} b\right\|_{H S}<\frac{\varepsilon}{k}\|E\|_{H S}
$$

we conclude by (9.2) and induction on the length of the word $m$ that

$$
\left.\left\|\mathrm{u}(\mathrm{m})-\mathrm{v}^{\prime}(\mathrm{m})\right\|_{\mathrm{HS}} \leq \text { (length of } \mathrm{m}\right) \frac{\varepsilon}{\mathrm{k}}\|\mathrm{E}\|_{\mathrm{HS}}
$$

By (9.2), E commutes with all $v_{j}^{\prime}$, so that $\left(v^{\prime}(m)\right) E=v(m)$ for each word $m$. Thus by (9.3) and the Schwartz inequality for the Hilbert-Schmidt norm,

$$
\left|(u(m) E, E)_{H S}-(v(m) E, E)_{H S}\right|<\varepsilon\|E\|_{H S}^{2}, m \in F_{k} \cdot
$$

By (9.1) and the definition of $\tau_{q}$, this is what we seek.

We are now ready to construct an embedding of $N$ into $R^{\omega}$. The construction depends on the following extension-lemma of Pearcy and Ringrose 
([15], Lemma 1), which we record for the convenience of the reader. (In what follows,-WOT denotes closure in the weak operator topology).

9.2. LEMMA. Let $M_{i}$ be a von Neumann algebra, $\phi_{i}$ a faithful, normal, positive linear functional on $M_{i}$, and $A_{i}$ a *-subalgebra of $M_{i}$ such that $A_{i}{ }^{- \text {WOT }}=M_{i}, i=1,2$. Suppose that $\pi$ is an algebraic $*-i$ omorphism from $A_{1}$ onto $A_{2}$ and that $\phi_{2} \circ \pi=\phi_{1}$ on $A_{1}$. Then $\pi$ can be extended to a *-isomorphism $\tilde{\pi}$ of $M_{1}$ onto $M_{2}$ with $\phi_{2} \circ \tilde{\pi}=\phi_{1}$ on $M_{1}$.

9.3. LEMMA. For each $\omega \in \beta z_{+} \backslash z_{+}$, there exists a trace-preserving *-monomorphism of $\mathrm{N}$ into $\mathrm{R}^{\omega}$.

PROOF. Let $F_{\infty}$ denote the free group on countably many generators $\left\{g_{n}\right\}$. Let $\tau_{0}$ denote the normalized trace on $R$. Since $N$ acts separably, it contains a strongly dense sequence, and so we may find a sequence $\left\{u_{n}\right\}$ of unitary operators generating $\mathrm{N}$ as a von Neumann algebra. Finally, let $\mathrm{F}_{\mathrm{k}} \subseteq \mathrm{F}_{\infty}$ be the set of all words involving only $\mathrm{g}_{1}, \ldots, \mathrm{g}_{\mathrm{k}}$ and with length $\leq \mathrm{k}$. Using Lemma 9.1 and the hyperfiniteness of $R$, choose for each $k$ unitaries $v_{1}^{k}, \ldots, v_{k}^{k}$ in $R$ such that

$$
\left|\tau(\mathrm{u}(\mathrm{m}))-\tau_{0}\left(\mathrm{v}^{\mathrm{k}}(\mathrm{m})\right)\right|<\frac{1}{\mathrm{k}}, \mathrm{m} \in \mathrm{F}_{\mathrm{k}} \text {, }
$$

where $v^{k}=\left(v_{1}^{k}, \ldots, v_{k}^{k}, I, I, \ldots\right)$. For each $j \in Z_{+}$, let $v_{j}$ be the unitary in $R^{\omega}$ represented by $\left(v_{j}{ }^{k}\right)_{k \in Z_{+}}$. Then since each $m \in F_{\infty}$ belongs to $F_{k}$ for all $k$ sufficiently large, we get, with $v=\left(v_{1}, \ldots, v_{j}, \ldots\right)$,

$$
\tau_{o, \omega}(\mathrm{v}(\mathrm{m}))=\lim _{\mathrm{k} \rightarrow \omega} \tau_{\mathrm{O}}\left(\mathrm{v}^{\mathrm{k}}(\mathrm{m})\right)=\tau(\mathrm{u}(\mathrm{m})), \mathrm{m} \in \mathrm{F}_{\infty} \text {. }
$$

Let $A=*$-algebra generated by $\left\{u_{n}\right\}$. For $x=\Sigma \lambda_{m} u(m)$, let $\pi(x)=\Sigma \lambda_{m} v(m)$. If $\Sigma \lambda_{m} u(m)=0$, then

$$
\sum \bar{\lambda}_{m} \lambda_{m^{\prime}} \tau\left(u(m) * u\left(m^{\prime}\right)\right)=0,
$$

whence by $(9.5)$

$$
\sum \lambda_{m} \lambda_{m^{\prime}} \tau_{0, \omega}\left(v(m) * v\left(m^{\prime}\right)\right)=0
$$

and so $\sum \lambda_{\mathrm{m}} \mathrm{v}(\mathrm{m})=0$. Thus $\pi$ is well-defined. By construction $\pi$ is a 
*-homomorphism of $A$ into $R^{\omega}$ which preserves the trace, and is hence monomorphic. We now apply Lemma 9.2 to $\pi$ to obtain the desired *-monomorphism of $\mathrm{N}$ into $\mathrm{R}^{\omega}$

9.4. LEMMA. For any $\omega \in \beta \mathrm{Z}_{+} \backslash \mathrm{Z}_{+}$there is trace-preserving *-monomorphism $\theta \quad$ of $N \otimes N$ into $(N \otimes R)^{\omega}$ such that

(a) For each $x \in N, \theta\left(x \otimes 1_{N}\right)$ is represented by the constant sequence $\left(x \otimes 1_{R}\right)_{\nu \in Z_{+}}$

(b) For each $y \in N, \theta\left(1_{N} \otimes y\right)$ is represented by a sequence of the form $\left(1_{N} \otimes z_{\nu}\right)_{\nu \in Z_{+}}, z_{\nu} \in R$

PROOF. Let $\pi_{1}$ be the unique isomorphism of $N$ into $(N \otimes R)^{\omega}$ such that $\pi_{1}(x)=\left(x \otimes 1_{R}\right)_{n} \in Z_{+} . x \in N, \pi$ the isomorphism of Lemma 9.3 , and $\pi_{2}$ the isomorphism of $N$ into $(N \otimes R)^{\omega}$ such that $\pi_{2}(y)=\left(1_{N} \otimes \pi(y)_{n}\right) n_{n} \in z_{+}$, where $\left(\pi(y)_{n}\right)_{n} \in z_{+}$is a representing sequence for $\pi(y)$ in $R^{\omega}, y \in N$. We claim that $\mathrm{x} \otimes \mathrm{y} \stackrel{\theta}{\rightarrow} \pi_{1}(\mathrm{x}) \pi_{2}$ (y) extends to a trace-preserving *-monomorphism $\quad \theta: N \otimes N \rightarrow(N \otimes R)^{\omega}$ satisfying (a) and (b).

With this definition, it is clear that $\theta$ satisfies (a) and (b), so we must show that $\theta$ is we1l-defined and can be extended as claimed.

Now $\pi_{1}$ and $\pi_{2}$ are $*$-isomorphisms, and so $\pi_{1} \otimes \pi_{2}$ defines a *-isomorphism on $\underset{\min }{\mathrm{N} \otimes \mathrm{N}}$. Since $\pi_{1}(\mathrm{~N})$ and $\pi_{2}(\mathrm{~N})$ are commuting subfactors of $(\mathrm{N} \otimes \mathrm{R})^{\omega}, \pi_{2}(\mathrm{~N}) \subseteq \pi_{1}(\mathrm{~N})^{\prime}$, so if $\eta$ denotes the $\eta$-isomorphism of Murray-von Neumann ( Sec. 6 ) we have $\theta=\eta \circ\left(\pi_{1} \otimes \pi_{2}\right)$ N $\theta \mathrm{N} \cdot \theta$ is thus a

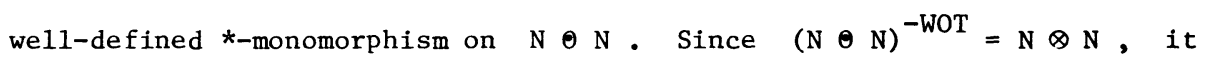
suffices by Lemma 9.2 to show that $\theta$ preserves the trace.

$$
\text { Let } \begin{aligned}
\tau=\text { trace on } N, \tau_{0} & =\text { trace on } R \text {. Then } \\
\left(\tau \otimes \tau_{0}\right)_{\omega}(\theta(x \otimes y)) & =\left(\tau \otimes \tau_{0}\right)_{\omega}\left[\left(x \otimes \pi(y)_{n}\right)\right] \\
& =\underset{n \rightarrow \omega}{\lim } \tau(x) \tau_{0}\left(\pi(y)_{n}\right)
\end{aligned}
$$




$$
\begin{aligned}
& =\tau(x) \lim _{n \rightarrow \omega} \tau_{0}\left(\pi(y)_{n}\right)=\tau(x) \tau_{0, \omega}(\pi(y)) \\
& =\tau(x) \tau(y)=\tau \otimes \tau(x \otimes y), \forall x, y \in N,
\end{aligned}
$$

i.e., $\theta$ preserves the trace.

9.5. LEMMA. Let $x_{1}, \ldots, x_{n} \in N, \varepsilon>0$. Then there exist $z_{1}, \ldots, z_{n} \in R$ and a unitary $x \in N \otimes R$ such that

$$
\left\|x_{j} \otimes 1_{R}-X\left(1_{N} \otimes z_{j}\right) X\right\|_{2}<\varepsilon, j=1, \ldots, n
$$

PROOF. Let $\theta: N \otimes N \rightarrow(N \otimes R)^{\omega}$ be the isomorphism of Lemma 9.4. As we

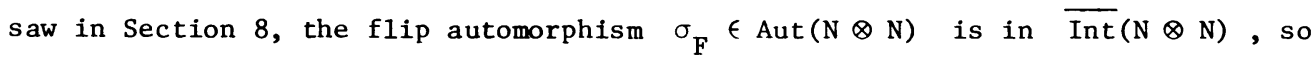
there is a unitary $v \in N \otimes N$ such that

$$
\left\|x_{j} \otimes 1_{N}-v\left(1_{N} \otimes x_{j}\right) v *\right\|_{2}<\frac{\varepsilon}{2}, j=1, \ldots, n \text {. }
$$

Now $\theta$ preserves the trace, and hence the $\mathrm{L}^{2}$-norm. Thus by (9.7),

$$
\left\|\theta\left(x_{j} \otimes 1\right)-\theta(v) \theta\left(1 \otimes x_{j}\right) \theta(v) *\right\|_{2}<\frac{\varepsilon}{2}, j=1, \ldots, n .
$$

Let $\left(\mathrm{X}_{\nu}\right)_{\nu \in \mathrm{Z}_{+}}$be a representing sequence of unitary operators in $\mathrm{N} \otimes \mathrm{R}$ for $\mathrm{X}=\theta(\mathrm{v}) \in(\mathrm{N} \otimes \mathrm{R})^{\omega}$ (the injectivity of $\theta$ is invoked here!). For each $\mathrm{j}$, let $\left(z_{j}^{(v)}\right)_{v \in z_{+}}$be a sequence in $R$ such that $\left(1 \otimes z_{j}{ }^{(U)}\right)$ represents $\theta\left(1 \otimes x_{j}\right)$. Then by $(9.8)$,

$$
\lim _{v \rightarrow \omega}\left\|x_{j} \otimes 1_{R}-x_{v}\left(1_{N} \otimes z_{j}^{(v)}\right) x_{v}\right\|_{2}<\frac{\varepsilon}{2}, j=1, \ldots, n \text {. }
$$

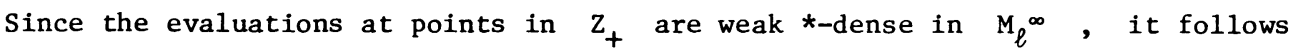
from (9.9) that $x_{v}$ and $z_{1}{ }^{(u)}, \ldots, z_{n}{ }^{(u)}$ satisfy $(9.6)$ for suitable $\nu \in z_{+}$. 10. THE PROOF OF THEOREM 8.1 COMPLETED.

Recall from Section 2 that a finite factor $M$ satisfies condition $\underline{C}$ if for each finite subset $\left\{x_{1}, \ldots, x_{n}\right\}$ of $M$ and $\varepsilon>0$, there exists a finitedimensional subfactor $C$ of $M$ and $v_{1}, \ldots, v_{n}$ in $C$ such that $\left\|x_{j}-v_{j}\right\|_{2}<\varepsilon, j=1, \ldots, n$. In order to show that $N$ is isomorphic to $R$, 
it suffices by Theorem 2.1 to verify condition $C$ for $N$. This is what we now proceed to do.

We saw that $N \cong N \otimes R$ in Section 8 . Now $R \cong M_{2} \otimes M_{2} \otimes \ldots$, where $M_{2}=2 \times 2$ matrices and the infinite tensor product is taken relative to the product state $\phi=\underset{n}{\otimes} \phi_{n}$, where each $\phi_{n}$ is the normalized trace on $M_{2}$. It follows that $R \cong R \otimes R \otimes \ldots$. , where the infinite tensor product is taken relative to the infinite tensor product of the canonical trace on $R$ (see [13], pp. 205-206). Hence we may identify $N$ with $N \otimes R \otimes R \otimes \ldots$. It follows from the definition of the infinite tensor product that $N \otimes R \otimes R \otimes \ldots$ contains an increasing sequence $\left\{\mathrm{N}_{\mathrm{k}}\right\}$ of subfactors such that

$$
N_{k} \cong N \otimes \underbrace{R \otimes \ldots \otimes R}_{k \text { times }}
$$

The relative commutant $\mathrm{N}_{\mathrm{k}}^{\mathrm{c}}$ of $\mathrm{N}_{\mathrm{k}}$ is $\mathrm{N}$ is isomorphic to $\mathrm{R} \otimes \mathrm{R} \otimes \ldots \cong \mathrm{R}$. Thus $N \otimes R \otimes R \otimes \ldots=N_{k} \otimes N_{k}^{c}$. Also, $\left\{N_{k}\right\}$ is an increasing sequence in $N \otimes R \otimes R \otimes \ldots$ such that $\bigcup_{k}^{U} N_{k}$ is $L^{2}$-dense, and $N_{k} \cong N \otimes R \otimes \ldots \otimes R \cong N$ for all $k$.

$k$ times

By the foregoing discussion, we may choose an increasing sequence $\left\{M_{k}\right\}$ of subfactors of $\mathrm{N}$ with the following properties:

(i) $\mathrm{M}_{\mathrm{k}} \cong \mathrm{N}$, for all $\mathrm{k}$.

(ii) $\bigcup_{k} M_{k}$ is $L^{2}$-dense in $N$.

(iii) The relative commutant $M_{k}^{c}$ of $M_{k}$ in $N$ is isomorphic to $R$, for a11 $\mathrm{k}$.

(iv) $\mathrm{N} \cong \mathrm{M}_{\mathrm{k}} \otimes \mathrm{M}_{\mathrm{k}}^{\mathrm{c}}$, for all $\mathrm{k}$.

Now, let $x_{1}, \ldots, x_{n} \in N$. By (ii), choose $k$ and $x_{1}{ }^{\prime}, \ldots, x_{n}^{\prime} \in M_{k}$ such that

$$
\left\|x_{j}-x_{j}\right\|_{2}<\frac{\varepsilon}{3}, j=1, \ldots, n \text {. }
$$

By (i) and (iii), there are isomorphisms 


$$
\theta_{1}: M_{k} \rightarrow N, \quad \theta_{2}: M_{k}^{c} \rightarrow R,
$$

So we may extend $\theta_{1} \otimes \theta_{2}$ to an isomorphism of $M_{k} \otimes M_{k}^{c} \rightarrow N \otimes R$ which preserves the trace (see the introduction), and hence the $\mathrm{L}^{2}$-norms. Thus, by identifying $M_{k}$ with its image under the isomorphism $a \rightarrow_{1}(a) \otimes 1_{R}, a \in M_{k}$, we may assume by (iv) that each $x_{j}^{\prime}$ has the form $m_{j} \otimes 1_{R}$ in some factorization of $N=M \otimes R, M \cong N$. By Lemma 9.5, there exist $z_{1}, \ldots, z_{n} \in R$ and a unitary $X \in M \otimes R$ such that

$$
\left\|x_{j}^{\prime}-x\left(1_{N} \otimes z_{j}\right) X\right\|_{2}<\frac{\varepsilon}{3}, j=1, \ldots, n
$$

Since $R$ is hyperfinite, there is a finite-dimensional subfactor $Q$ of $R$ and $q_{1}, \ldots, q_{n} \in Q$ such that

$$
\left\|z_{j}-q_{j}\right\|_{2}<\frac{\varepsilon}{3}, j=1, \ldots, n
$$

Then $X(C \otimes Q) X^{*} \quad$ is a finite-dimensional subfactor of $N=M \otimes R$, and by $(10.1)-(10.3)$

$$
\left\|x_{j}-X\left(1_{M} \otimes q_{j}\right) X *\right\|_{2}<\varepsilon, j=1, \ldots, n
$$

This verifies condition $\mathrm{C}$ for $\mathrm{N}$.

11. CLASSIFICATION OF INJECTIVE FACTORS OF TYPE $\operatorname{II}_{\infty}$, $\operatorname{III}_{\lambda}, \lambda \in[0,1)$.

With Theorem 8.1 and the fundamental structure Theorems $2.2,2.3$, and 2.4 now at our disposal, the precise structure of injective factors of type II $_{\infty}$ and $\operatorname{III}_{\lambda}, \lambda \in(0,1]$ can now be deduced in a fairly straightforward fashion.

Recall from Section 2 that $R_{0,1}$ is the hyperfinite $I_{\infty}$ factor $R \otimes B(H)$, where $H$ is a separable Hilbert space.

11.1. THEOREM ([10], Theorem 7.4). All separably acting injective factors of type $I I_{\infty}$ are isomorphic to $R_{0,1}$.

PROOF. Let $M$ be an injective $I_{\infty}$ factor acting on a separable Hilbert space $H$ - By [4], Theorem IX, we can write $M \cong N \otimes B(H)$ for a separably acting $I_{1}$ factor $N . M$ can thus be viewed as the algebra of $\infty \times \infty$ matrices 
with entries in $N$. The map $F:\left(x_{i j}\right) \in M \rightarrow x_{11}$ defines a projection of norm 1 of $M$ onto $\mathrm{N}$, and composing this with a projection of $B(H \otimes H)$ onto $M$, we see that $\mathrm{N}$ is injective. By Theorem 8.1, $\mathrm{N}$ is isomorphic to $\mathrm{R}$, whence $M$ is isomorphic to $\mathrm{R}_{0,1}$.

If $\left\{M_{\alpha}\right\}$ is an increasing family of injective von Neumann algebras, it follows by [14], Proposition 5.7 that the weak closure of $U_{\alpha} M_{\alpha}$ is injective. Since every finite-dimensional algebra is injective, we conclude that all hyperfinite von Neumann algebras are injective. Applying Theorem 11.1, we deduce

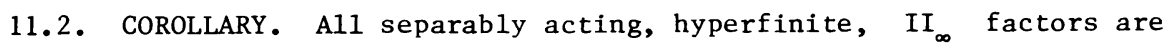
isomorphic.

11.3. THEOREM. ([10], Theorem 7.7). Let $\lambda \in(0,1)$. All separably acting injective factors of type $\operatorname{III}_{\lambda}$ are isomorphic to Powers factor $R_{\lambda}$. PROOF. Let $M$ be an injective $\operatorname{III}_{\lambda}$ factor acting on a separable Hilbert space with discrete decomposition $W^{*}(N, \theta)$ obtained via Theorem 2.3. We want to show that $\mathrm{N}$ is injective.

By Theorem 2.2 and [28], p. 303, we can find an abelian von Neumann algebra $A$ and a one-parameter action $a$ on $A \otimes N$ such that $M \cong W^{*}(A \otimes N, a)$. By [28], Theorem 8.1, the fixed point algebra of $\hat{a}$ on $M$ is precisely $A \otimes N$. Hence, if $\mu$ is an invarient mean on $\ell^{\infty}(R)$, it is straightforward to verify that

$$
(E(x) \xi, \eta)=\mu \hat{x a}(x) \xi, \eta)\}, x \in M, \xi, \eta \in H
$$

defines a projection $E$ of norm 1 of $M$ onto $A \otimes N$. Since $M$ is injective, we conclude that $A \otimes N$ is injective. Thus by [14], Proposition 5.6, $N$ is injective.

We conclude by Theorem 11.1 that $\mathrm{N}$ is isomorphic to $\mathrm{R}_{0,1}$. But by [9] Corollary 6 and [5], Théorème $4.4 .1, R_{\lambda}$ is the only $\operatorname{III}_{\lambda}$ factor the II $_{\infty}$ part of whose discrete decomposition is isomorphic to $R_{0,1}$. Hence $M$ is isomorphic to $R_{\lambda}$

11.4. THEOREM ([10], Theorem 7.5). If $M$ is a separably acting injective 
factor of type $\operatorname{III}_{0}$, then $M$ is a Krieger factor.

PROOF. Let $W^{*}(\mathrm{~N}, \theta)$ be a discrete decomposition of $M$ as in Theorem 2.4. As in the proof of Theorem 11.3, $\mathrm{N}$ is injective; however, it is not in general a factor. On the other hand, it is a direct integral of factors, and each factor appearing in its direct integral decomposition (except possibly for a set of measure 0 ) is injective by [10], Proposition 6.5 and of type II $_{\infty}$, and hence isomorphic to $R_{0,1}$ - But these are precisely the hypotheses of [8], Theorem II.1, which asserts that under these conditions $M$ is a Krieger factor QED

It follows from [30] that injective III $_{0}$ factors are classified up to isomorphism by ergodic non-transitive flows. For further details, the reader can consult [30], [10], and [37].

The only known injective factor of type III ${ }_{1}$ is the factor $R_{\infty}$ of Araki and Woods (see Section 2). It is not known whether this is the only possible one.

ACKNOWLEDGEMENTS. I would like to thank Sze-kai Tsui for several stimulating and instructive conversations about the material contained herein. I would also like to thank Jim Agler, John B. Conway, and Andrew Lenard for very active participation in a seminar given during the summer of 1978 at Indiana University, during which many inaccuracies in an early version of this work were detected and corrected.

\section{REFERENCES}

1. F.J. MURRAY and J. VON NEUMANN, On Rings of Operators, Ann. Math. 37 (1936) 116-229.

2. F.J. MURRAY and J. VON NEUMANN, On Rings of Operators, II Trans. Amer, Math. Soc. 41 (1937), 208-248.

3. J. VON NEUMANN, On Rings of Operators, III, Ann. Math. 41 (1940), 94-161.

4. F.J. MURRAY and J. VON NEUMANN, On Rings of Operators, IV Ann. Math. 44 (1943), 716-808.

5. A. CONNES, Une Classification des Facteurs de Type III, Ann. Sci. Ecole Norm. Sup. 6 (1973), 133-252.

6. M. TAKESAKI, Tomita's Theory of Modular Hilbert Algebras and its Applications, 
Lec, Notes Math. 128 (1970), Springer-Verlag, New York.

7. A. CONNES, Almost Periodic States and Factors of Type III 1 J. Func. Anal. 16 (1974), 415-445.

8. A. CONNES, On Hyperfinite Factors of Type $\mathrm{III}_{0}$ and Krieger's Factors, J. Func. Anal. 18 (1975), 318-327.

9. A. CONNES, Outer Conjugacy Classes of Automorphisms of Factors, Ann. Sci. Ecole Norm. Sup. 8 (1975), 383-420.

10. A. CONNES, Classification of Injective Factors, Ann. Math. 104 (1976), 73-115.

11. D. TOPPING, Lectures on von Neumann Algebras, Van Nostrand Rheinhold Math. Studies $36(1971)$, Van Nostrand, London.

12. J. DIXMIER, Les Algebras d'operateurs Dans 1'espace Hilbertien, 1969, Gauthier-Villars, Paris.

13. S. SAKAI, $\mathrm{C}^{*-a l g e b r a s}$ and $\mathrm{W}^{*}$-algebras, 1971, Springer-Verlag, New York.

14. E. EFFROS, and E.C. LANCE, Tensor Products of Operator Algebras, Adv. Math. 25 (1977), 1-33.

15. C. PEARCY, and J.R. RINGROSE, Trace-preserving Isomorphisms in Finite Operator Algebras, Amer. J. Math. 90 (1968), 444-455.

16. J. VON NEUMANN, On Rings of Operators: Reduction Theory, Ann. Math. 50 (1949), 401-485.

17. L. PUKANSKY, Some Examples of Factors, Pub1. Math. 4 (1956), 135-156.

18. J.T. SCHWARTZ, Two Finite, Non-hyperfinite, Nonisomorphic Factors, Commun. Pure App1. Math. 16 (1963), 19-26.

19. J.T. SCHWARTZ, Non-isomorphism of a Pair of Factors of Type III, Commun, Pure Appl. Math. 16 (1963), 111-120.

20. R.T. POWERS, Representations of Uniformly Hyperfinite Algebras and Their Associated von Neumann Rings, Ann. Math. 86 (1967), 138-171.

21. D. McDUFF Uncountably Many II Factors, Ann. Math. 90 (1969), 372-377.

22. S. SAKAI, An Uncountable Family of Non-hyperfinite Type III Factors, Functional Analysis, Academic Press, New York, 1970, 65-70.

23. S. SAKAI, An Uncountable Number of II $_{1}$, II I $_{\infty}$ Factors J. Func. Anal. 5 (1970), 236-246.

24. H. ARAKI ant E.J. WOODS, A Classification of Factors, Pub1. RIMS Kyoto Univ. $4(1968), 51-130$.

25. M. TOMITA, Standard Forms of von Neumann Algebras, 5th Func. Anal. Symp. Math. Soc. Japan, Sendai, 1967.

26. M. TOMITA, Quasi-standard von Neumann Algebras, mimeographed notes, 1967.

27. M. RIEFFEL and A. VAN DAELE, A Bounded Operator Approach to Tomita-Takesak1 Theory, Pacific J. Math. 69 (1977), 187-221. 
28. M. TAKESAKI, Duality in Cross Products and the Structure of von Neumann Algebras of Type III, Acta Math. 131 (1973), 249-310.

29. W.B. ARVESON, On Groups of Automorphisms of Operator Algebras, J. Func. Anal. 15 (1974), 217-243.

30. W. KRIEGER, On Ergodic Flows and Isomorphism of Factors, Math. Ann. 223 $(1976), 19-70$.

31. J. TOMIYAMA, On the Projection of Norm One in $W^{*-a l g e b r a s, ~ P r o c . ~ J a p a n ~ A c a d . ~}$ 33 (1957), 608-612.

32. I.E. SEGAL, A Non-commutative Extension of Abstract Intergration, Ann. Math. 57 (1953), 401-457.

33. M. DAY, Amenable Semigroups, Illinois J. Math. 1 (1957), 509-544.

34. K.-F. NG and Y.-C. WONG, Partially Ordered Topological Vector Spaces, 1973, Oxford University Press, Oxford.

35. E. FØLNER, On Groups With Full Banach Mean Value, Math. Scand. 3 (1955), 243-254.

36. R.T. POWERS and E. ST $\emptyset$ RMER, Free States of the Canonical Anti-commutation Relations, Commun. Math. Phys. I6 (1970), 1-33.

37. A. CONNES, On the Classification of von Neumann Algebras and Their Automorphisms, Symp. Math., Ist. Nationale di Alta Mat. 20 (1976), 435-478.

38. S. SAKAI, The Theory of $\mathrm{W}^{*}$-algebras, Yale University mimeographed lecture notes, 1962 .

39. M. TAKESAKI, On the Cross-Norm of the Direct Product of C*-algebras, Tohoku Math. J. 16 (1964), 111-122.

40. R. KALlMAN, A Generalization of Free Action, Duke Math. J. 36 (1969), $781-789$.

41. D. McDUFF, Central Sequences and the Hyperfinite Factor, Proc. London Math. Soc. 21 (1970), 443-461. 


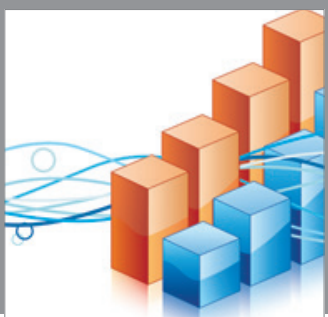

Advances in

Operations Research

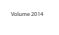

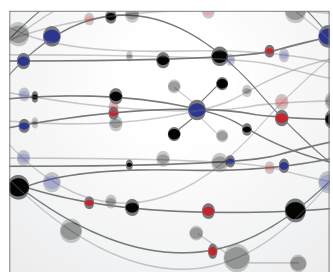

\section{The Scientific} World Journal
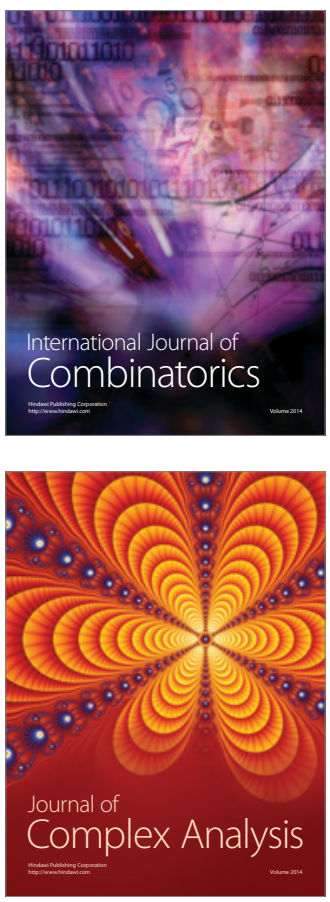

International Journal of

Mathematics and

Mathematical

Sciences
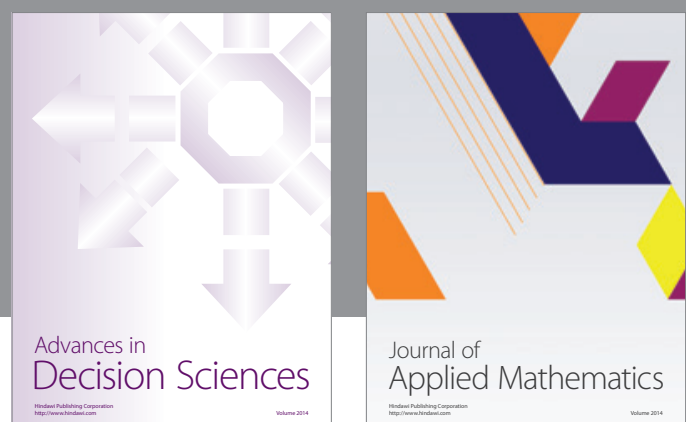

Journal of

Applied Mathematics
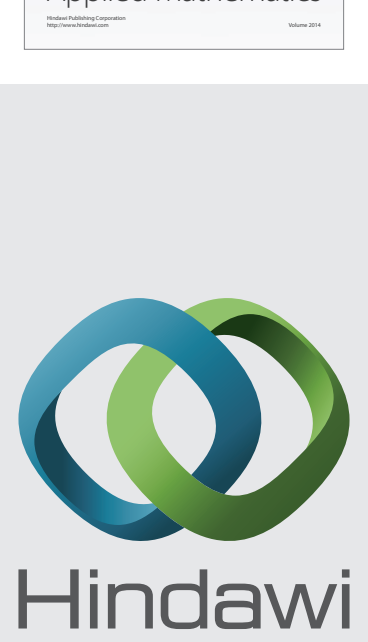

Submit your manuscripts at http://www.hindawi.com
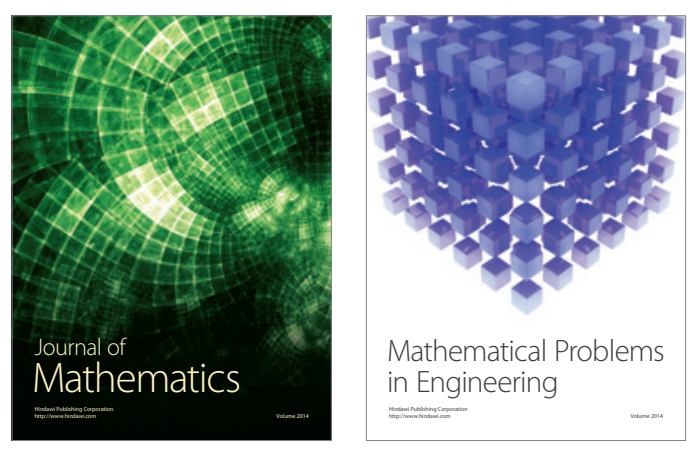

Mathematical Problems in Engineering
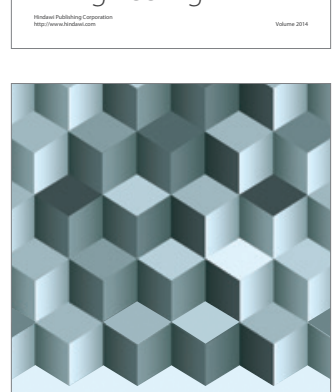

Journal of

Function Spaces
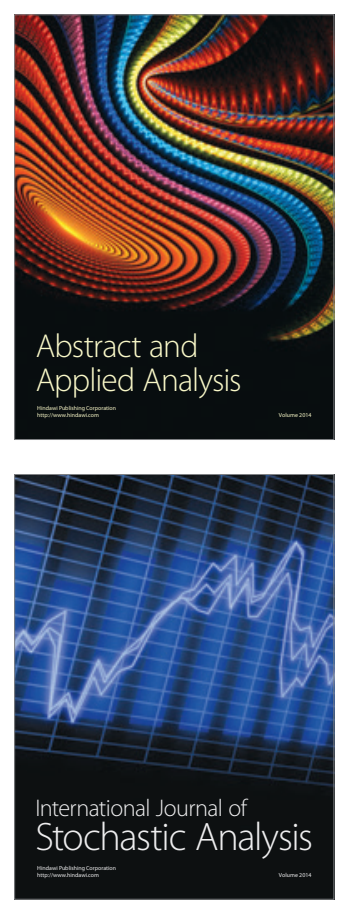

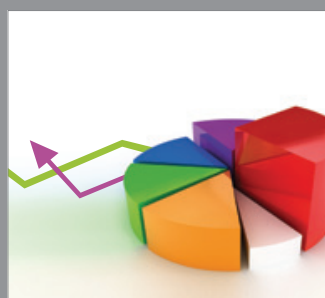

ournal of

Probability and Statistics

Promensencen
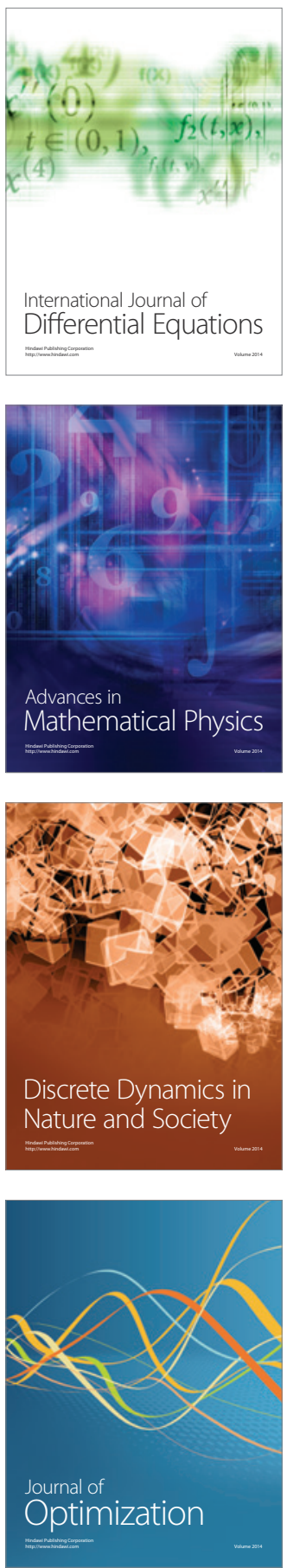